\title{
Comparative analysis of thylakoid protein complexes in state transition mutants nsi and stn7: focus on PSI and LHCII
}

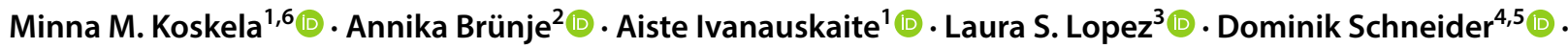

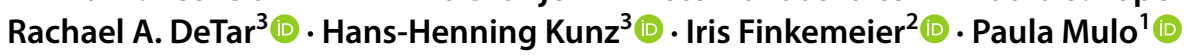

Received: 7 July 2019 / Accepted: 9 January 2020 / Published online: 23 January 2020

(c) The Author(s) 2020

\begin{abstract}
The photosynthetic machinery of plants can acclimate to changes in light conditions by balancing light-harvesting between the two photosystems (PS). This acclimation response is induced by the change in the redox state of the plastoquinone pool, which triggers state transitions through activation of the STN7 kinase and subsequent phosphorylation of light-harvesting complex II (LHCII) proteins. Phosphorylation of LHCII results in its association with PSI (state 2), whereas dephosphorylation restores energy allocation to PSII (state 1). In addition to state transition regulation by phosphorylation, we have recently discovered that plants lacking the chloroplast acetyltransferase NSI are also locked in state 1, even though they possess normal LHCII phosphorylation. This defect may result from decreased lysine acetylation of several chloroplast proteins. Here, we compared the composition of wild type (wt), $\sin 7$ and $n s i$ thylakoid protein complexes involved in state transitions separated by Blue Native gel electrophoresis. Protein complex composition and relative protein abundances were determined by LC-MS/MS analyses using iBAQ quantification. We show that despite obvious mechanistic differences leading to defects in state transitions, no major differences were detected in the composition of PSI and LHCII between the mutants. Moreover, both $\sin 7$ and $n s i$ plants show retarded growth and decreased PSII capacity under fluctuating light as compared to wt, while the induction of non-photochemical quenching under fluctuating light was much lower in both $n s i$ mutants than in $\sin 7$.
\end{abstract}

Keywords Arabidopsis $\cdot$ Light-harvesting complex $\cdot$ Lysine acetylation $\cdot$ State transitions $\cdot$ Photosystem I

Minna M. Koskela and Annika Brünje have contributed equally to this work

Electronic supplementary material The online version of this article (https://doi.org/10.1007/s11120-020-00711-4) contains supplementary material, which is available to authorized users.

Iris Finkemeier

iris.finkemeier@uni-muenster.de

$\triangle$ Paula Mulo

pmulo@utu.fi

1 Department of Biochemistry, Molecular Plant Biology, University of Turku, Biocity A, Tykistökatu 6, 20520 Turku, Finland

2 Plant Physiology, Institute of Plant Biology and Biotechnology, University of Münster, Schlossplatz 7, 48149 Münster, Germany

\section{Introduction}

Light is an important environmental signal affecting most aspects of plant life including germination, phototropism and flowering. Additionally, light is the driving force of photosynthetic electron transfer and carbon assimilation, which provide plants with the energy needed for their growth and development. The light-harvesting complexes (LHCs), composed of chlorophyll and other pigment molecules bound to

3 Plant Physiology, School of Biological Sciences, Washington State University, Pullman, WA 99164-4236, USA

4 Compact Plants Phenomics Center, Washington State University, Pullman, WA 99164, USA

5 Institute of Biological Chemistry, Washington State University, Pullman, WA 99164-6340, USA

6 Present Address: Centre Algatech, Institute of Microbiology, Czech Academy of Sciences, Novohradská 237 - Opatovický mlýn, 37981 Třebon, Czech Republic 
the thylakoid-embedded LHC proteins, absorb light energy and funnel it towards the reaction centers P680 in Photosystem II (PSII) and P700 in Photosystem I (PSI). Excitation of the special pair of reaction center chlorophylls is followed by charge separation, splitting of water and electron transfer through the redox-active components of the thylakoid membrane, which finally leads to the reduction of NADP ${ }^{+}$ to NADPH. The electron transfer is accompanied by proton translocation into the thylakoid lumen, and the resulting proton gradient is harnessed by the ATP synthase for ATP production. Subsequently, NADPH and ATP are used in the Calvin-Benson cycle for $\mathrm{CO}_{2}$ assimilation. Importantly, several enzymes functioning in carbon assimilation are also redox activated according to ambient illumination (Buchanan and Balmer 2005).

Plants have developed several systems to sense changes in ambient illumination, such as the red and blue light-sensing light receptor molecules, phytochromes and phototropins, respectively. However, the photosynthetic machinery can sense changes in light quality and intensity also directly via the redox state of the plastoquinone (PQ) pool, which triggers appropriate long- and short-term responses for balancing the redox poise between the two photosystems. The redox state of the PQ pool is known to regulate gene expression both in the chloroplasts and the nucleus, which results in stoichiometry adjustments of the photosystems to meet the needs of the plant under new environmental conditions in the long term (Steiner et al. 2009; Puthiyaveetil et al. 2010, 2012). Short-term acclimation is achieved through thermal dissipation of absorbed excess energy (non-photochemical quenching (NPQ)) in seconds, and via state transitions, i.e. re-equilibration of the absorption cross-section of PSII and PSI within the time scale of minutes (Bonaventura and Myers 1969; Murata 1969). LHCII is an important player in these acclimation processes, as it functions both in harvesting and dissipation of light energy depending on ambient conditions (Rochaix 2014). State transitions are mediated through the phosphorylation and dephosphorylation of LHCII proteins: phosphorylation of LHCII results in association of LHCII to PSI (state 2), while LHCII dephosphorylation leads to reallocation of energy to PSII (state 1) (Bennett 1977; Pietrzykowska et al. 2014). Phosphorylation of the major LHCII antenna proteins, LHCB1 and LHCB2, is catalyzed by the STN7 kinase, which is activated upon docking of $\mathrm{PQH}_{2}$ to the $\mathrm{Q}_{\mathrm{o}}$ site of the Cyt $\mathrm{b}_{6}$ f complex (Bennett 1977; Allen et al. 1981; Depège et al. 2003; Bellafiore et al. 2005). On the other hand, over-excitation of PSI results in the oxidation of the PQ pool, deactivation of STN7 and dephosphorylation of LHCII by the TAP38/PPH1 phosphatase (Pribil et al. 2010; Shapiguzov et al. 2010).

The composition and phosphorylation patterns of lightharvesting complexes under various conditions have been well studied in numerous phototrophic organisms. In
Arabidopsis thaliana (hereafter Arabidopsis), LHCII is composed of LHCB1-LHCB6 proteins (Jansson 1999). LHCB1LHCB3 form the trimeric LHCII antenna, which may be either strongly (S-LHCII composed of LHCB1 and LHCB2), moderately (M-LHCII composed of LHCB1 and LHCB3), or loosely (L-LHCII composed of mainly LHCB1 and LHCB2) bound to PSII. The S-trimer is bound to the PSII core monomer via minor antenna protein LHCB5, whereas the M-trimer is associated to the core via LHCB4 and LHCB6 proteins. Due to the very loose interaction, the binding site of the L-trimer is still elusive (Dekker and Boekema 2005; Kouril et al. 2005; Galka et al. 2012). Approximately, half of the LHC protein pool at the thylakoids form the so-called "extra" or "free" L-LHCII trimers, which are not tightly bound to the photosystems, but serve as a shared antenna for both PSII and PSI (Grieco et al. 2012; Wientjes et al. 2013). LHCI, in turn, is composed of LHCA1-LHCA4 proteins, which are surrounding PSI in the shape of a half-moon (Boekema et al. 2001; Ben-Shem et al. 2003). Upon phosphorylation of LHCB2 protein, a portion of L-LHCII becomes associated with PSI either through the PSAH/PSAI/PSAL/PSAO proteins and/or through the LHCA antenna (Lunde et al. 2000; Galka et al. 2012; Crepin and Caffarri 2015; Longoni et al. 2015; Benson et al. 2015; Pan et al. 2018).

We have recently identified Arabidopsis mutant lines devoid of the chloroplast lysine acetyltransferase NSI, which similar to the $\sin 7$ loss-of-function mutant, are not capable of forming the PSI-LHCII complex and thus performing state transitions (Depège et al. 2003; Bellafiore et al. 2005; Koskela et al. 2018). Intriguingly, no differences in the pattern of LHCII phosphorylation were detected between the $n s i$ and wt plants, but the $n s i$ plants possessed decreased lysine acetylation of several chloroplast proteins as compared to wt (Koskela et al. 2018). Hence, the mechanisms leading to defects in state transitions in the $\sin 7$ and $n s i$ mutants must be fundamentally different. To get a deeper insight into these mechanistic differences, we compared the composition of the protein complexes involved in state transitions (i.e. PSI complex and LHCII trimers) using large pore (lp) Blue Native (BN) gel electrophoresis for protein complex separation and LC-MS/MS for analyzing specific protein complexes excised from the gel. Moreover, we have compared the growth and photosynthetic performance of $\operatorname{stn} 7$ and $n s i$ plants with fluctuating light treatment, which has previously been shown to have a strong effect on the growth of $\operatorname{stn} 7$ (Bellafiore et al. 2005; Tikkanen et al. 2010).

\section{Material and methods}

\section{Plant material}

For lpBN gel electrophoresis and subsequent MS analysis, Arabidopsis thaliana wt plants (Col-0) and the T-DNA 
mutant lines nsi-1 (SALK_033944), nsi-2 (SALK_020577) (Koskela et al. 2018) and $\operatorname{stn} 7$ (SALK_073254) (Tikkanen et al. 2006) were grown in $8 \mathrm{~h}$ light/16 h darkness at photosynthetic photon flux density (PPFD) of $100 \mu \mathrm{mol} \mathrm{m} \mathrm{m}^{-2} \mathrm{~s}^{-1}$, $50 \%$ humidity and $+23{ }^{\circ} \mathrm{C}$. For physiological experiments, plants were grown in $12 \mathrm{~h}$ light/12 h darkness at PPFD of $90 \mu \mathrm{mol}$ photons $\mathrm{m}^{-2} \mathrm{~s}^{-1}$ for 2 weeks followed by a shift to fluctuating light (cycles of $4 \mathrm{~min} 90 \mu \mathrm{mol} \mathrm{m} \mathrm{m}^{-2} \mathrm{~s}^{-1}$ followed by $1 \mathrm{~min} 900 \mu \mathrm{mol} \mathrm{m}^{-2} \mathrm{~s}^{-1}$ ) for 2 weeks (Schneider et al. 2019).

\section{Growth curves and fluorescence measurements}

Photosynthesis-related parameters and growth curves were determined with daily induction curves on dark-adapted plants using a MAXI version IMAGING-PAM (IMAG-K7 by Walz, Effeltrich, Germany). Plants were dark-adapted for $20 \mathrm{~min}$ before the induction curve was measured for $300 \mathrm{~s}$ at $186 \mu \mathrm{mol} \mathrm{m}{ }^{-2} \mathrm{~s}^{-} 1$ with saturating pulses given every $20 \mathrm{~s}$. The reported Y(II), NPQ and Y(NO) values were calculated by averaging the last two data points of the induction curve. Data analysis of the 15-day light-treatment experiment were carried out using the ImagingPAMProcessing toolkit (Schneider et al. 2019).

\section{Determination of chlorophyll content}

Chlorophyll determination was done as described in (Porra et al. 1989). In brief, rosette tissue from plants grown as described above for physiological experiments was harvested individually at 4.5 weeks of age. Tissue was ground in liquid $\mathrm{N}_{2}$, and chlorophyll was extracted by incubating $10-20 \mathrm{mg}$ of material in $1.5 \mathrm{~mL}$ of ice cold $80 \%$ acetone for $2 \mathrm{~h}$ on ice. Samples were spun at 15,000 rpm for $5 \mathrm{~min}$, then supernatant was measured in a spectrophotometer at 646, 663 and $750 \mathrm{~nm}$. For statistical analysis, a Shapiro-Wilk test was used to determine if data was normally distributed. If data had a normal distribution, a 2-way ANOVA and a series of Tukey's multiple comparison tests were used to determine if means were statistically different. If data was not normally distributed, a Kruskal-Wallis test with a series of Dunn's multiple comparison tests were used to determine if means were statistically different.

The chlorophyll concentration of intact thylakoids, or thylakoids solubilized with digitonin or DM (Järvi et al. 2011) was determined as described previously (Porra et al. 1989).

\section{Extraction of thylakoid proteins}

Fresh Arabidopsis leaves were ground in $300 \mathrm{mM}$ sucrose, $50 \mathrm{mM}$ HEPES-KOH pH 7.6, $5 \mathrm{mM} \mathrm{MgCl}_{2}, 1 \mathrm{mM}$ NaEDTA, $1.25 \%$ BSA, $22 \mathrm{mM}$ ascorbate, and $10 \mathrm{mM} \mathrm{NaF}$. The homogenate was filtered through pre-soaked Miracloth
(Millipore) and the filtrate was centrifuged for $4 \mathrm{~min}$, $4000 \times g,+4{ }^{\circ} \mathrm{C}$. Chloroplasts were lysed by resuspending the pellet in a hypotonic lysis buffer $(5 \mathrm{mM}$ sucrose, $10 \mathrm{mM}$ Hepes-KOH pH 7.6, $5 \mathrm{mM} \mathrm{MgCl} 2,10 \mathrm{mM} \mathrm{NaF}$, Pierce ${ }^{\mathrm{TM}}$ protease inhibitor (Thermo Scientific)). The lysate was centrifuged at $18,000 \times g$ for $5 \mathrm{~min},+4{ }^{\circ} \mathrm{C}$, and the pellet was resuspended in $100 \mathrm{mM}$ sucrose, $10 \mathrm{mM}$ Hepes-KoH pH 7.6, $10 \mathrm{mM} \mathrm{MgCl}_{2}$, and $10 \mathrm{mM} \mathrm{NaF}$ for storage in $-80^{\circ} \mathrm{C}$. Different biological replicates were prepared from plants grown at different times on separate trays.

\section{Native and 2D gel electrophoresis, trypsin-digestion, LC-MS/MS and data analysis}

LpBN gels and samples were prepared as previously described (Järvi et al. 2011). Protein complexes were excised from the lpBN gels, trypsin digested (Morgan et al. 2008), and analyzed using LC-MS/MS as described in (Koskela et al. 2018). The raw spectrum files of all replicates from the individual protein complexes were processed together using the MaxQuant software version 1.5.2.8 (https://www.maxqu ant.org/) (Cox and Mann 2008; Tyanova et al. 2016), with match between runs and intensity-based absolute quantification (iBAQ) enabled, and peptide and protein FDR $<1 \%$. The spectrum files were searched against the Araport 11 Arabidopsis protein fasta database, a reverse decoy database, and a standard contaminant list integrated into MaxQuant. Lysine acetylation, protein $\mathrm{N}$-term acetylation and methionine oxidation were searched as variable modifications. The resulting 'protein groups' data files were further processed with Perseus version 1.6.1.3 (Tyanova et al. 2016). Reverse hits and contaminants were removed. iBAQ intensities were $\log 2$ transformed. Technical replicates were averaged and protein groups identified in only one replicate of each genotype were filtered out. Data were analyzed from three independent biological replicates. Protein abundances within each complex were reported as iBAQ values, which were normalized to PSAA (Tables 1 and 2) or LHCB3 (Table 3). The spectrum file of the LHCII trimer complex (digitonin solubilization) was re-analyzed from Koskela et al. (2018) (www.plantcell.org, copyright American Society of Plant Biologists).

For the 2D gel electrophoresis, lpBN gel strips were solubilized and run in a second dimension on $12 \%$ reducing SDS-PAGE supplemented with $6 \mathrm{M}$ urea (Suorsa et al. 2015). Gels were stained with SYPRO ${ }^{\mathrm{TM}}$ total protein stain according to the manufacturer's instructions.

\section{Statistical analyses for proteomics}

iBAQ values can be used to calculate the relative protein abundances of different proteins within one complex (Schwanhäusser et al. 2011). However, they do not allow 
Table 1 Quantitative proteome analysis according to iBAQ values of the proteins identified within the PSI/PSII bands excised from lpBN-PAGE gels

\begin{tabular}{|c|c|c|c|c|c|c|}
\hline \multicolumn{2}{|l|}{ Proteins/protein groups } & \multirow[t]{2}{*}{ Subunit of } & \multicolumn{4}{|l|}{ Genotype } \\
\hline Locus/loci & Name(s) & & wt & $n s i-1$ & $n s i-2$ & $\operatorname{stn} 7$ \\
\hline AT4G28750 & PSAE-1 & PSI & $1.58( \pm 0.05)$ & $1.46( \pm 0.14)$ & $1.49( \pm 0.26)$ & $1.61( \pm 0.38)$ \\
\hline AT4G02770 & PSAD-1 & PSI & $1.38( \pm 0.30)$ & $1.52( \pm 0.15)$ & $1.42( \pm 0.08)$ & $1.39( \pm 0.02)$ \\
\hline AT1G55670 & PSAG & PSI & $1.38( \pm 0.48)$ & $1.01( \pm 0.36)$ & $0.86( \pm 0.39)$ & $0.94( \pm 0.19)$ \\
\hline ATCG01060 & PSAC & PSI & $1.01( \pm 0.39)$ & $1.15( \pm 0.36)$ & $1.10( \pm 0.26)$ & $1.06( \pm 0.51)$ \\
\hline ATCG00350 & PSAA & PSI & 1.00 & 1.00 & 1.00 & 1.00 \\
\hline AT1G31330 & PSAF & PSI & $0.89( \pm 0.20)$ & $0.99( \pm 0.12)$ & $0.93( \pm 0.11)$ & $0.92( \pm 0.18)$ \\
\hline AT1G61520 & LHCA3 & PSI & $0.73( \pm 0.12)$ & $0.99( \pm 0.08)$ & $0.92( \pm 0.15)$ & $0.88( \pm 0.36)$ \\
\hline AT3G54890 & LHCA1 & PSI & $0.61( \pm 0.11)$ & $0.64( \pm 0.05)$ & $0.61( \pm 0.09)$ & $0.66( \pm 0.15)$ \\
\hline AT1G52230 & PSAH-2 & PSI & $0.58( \pm 0.28)$ & $0.56( \pm 0.17)$ & $0.67( \pm 0.21)$ & $0.78( \pm 0.06)$ \\
\hline AT3G47470 & LHCA4 & PSI & $0.58( \pm 0.19)$ & $0.82( \pm 0.09)$ & $0.80( \pm 0.03)$ & $0.81( \pm 0.33)$ \\
\hline ATCG00340 & PSAB & PSI & $0.50( \pm 0.13)$ & $0.63( \pm 0.11)$ & $0.59( \pm 0.03)$ & $0.62( \pm 0.09)$ \\
\hline AT4G12800 & PSAL & PSI & $0.45( \pm 0.07)$ & $0.64( \pm 0.07)$ & $0.56( \pm 0.06)$ & $0.57( \pm 0.13)$ \\
\hline AT1G30380 & PSAK & PSI & $0.31( \pm 0.03)$ & $0.38( \pm 0.01)$ & $0.36( \pm 0.03)$ & $0.32( \pm 0.17)$ \\
\hline AT3G61470; AT5G28450 & LHCA2; CAB family & PSI & $0.28( \pm 0.17)$ & $0.54( \pm 0.08)$ & $0.68( \pm 0.24)$ & $0.44( \pm 0.26)$ \\
\hline AT2G20260 & PSAE-2 & PSI & $0.27( \pm 0.08)$ & $0.27( \pm 0.02)$ & $0.27( \pm 0.05)$ & $0.29( \pm 0.03)$ \\
\hline AT5G64040 & PSAN & PSI & $0.13( \pm 0.04)$ & $0.15( \pm 0.02)$ & $0.18( \pm 0.04)$ & $0.17( \pm 0.09)$ \\
\hline AT2G46820 & PSAP & PSI & $0.03( \pm 0.02)$ & $0.04( \pm 0.02)$ & $0.03( \pm 0.02)$ & $0.03( \pm 0.01)$ \\
\hline AT1G08380 & PSAO & PSI & $0.01( \pm 0.02)$ & $0.03( \pm 0.02)$ & $0.03( \pm 0.01)$ & $0.02( \pm 0.02)$ \\
\hline ATCG00280 & CP43 (PSBC) & PSII & $0.24( \pm 0.09)$ & $0.16( \pm 0.04)$ & $0.12( \pm 0.01)$ & $0.16( \pm 0.04)$ \\
\hline AT1G29920; AT1G29910 & LHCB1.1; LHCB1.2* & LHCII & $0.24( \pm 0.06)$ & $0.14( \pm 0.05)$ & $0.11( \pm 0.04)$ & $0.09( \pm 0.04)$ \\
\hline ATCG00680 & CP47 (PSBB) & PSII & $0.20( \pm 0.07)$ & $0.14( \pm 0.02)$ & $0.10( \pm 0.03)$ & $0.14( \pm 0.03)$ \\
\hline ATCG00270 & D2 (PSBD) & PSII & $0.15( \pm 0.05)$ & $0.10( \pm 0.02)$ & $0.08( \pm 0.01)$ & $0.12( \pm 0.02)$ \\
\hline ATCG00020 & D1 (PSBA) & PSII & $0.10( \pm 0.05)$ & $0.07( \pm 0.01)$ & $0.05( \pm 0.01)$ & $0.06( \pm 0.01)$ \\
\hline AT4G10340 & CP26 (LHCB5) & PSII & $0.07( \pm 0.02)$ & $0.08( \pm 0.01)$ & $0.06( \pm 0.02)$ & $0.07( \pm 0.01)$ \\
\hline AT3G50820 & PSBO- $2 *$ & PSII & $0.07( \pm 0.01)$ & $0.04( \pm 0.01)$ & $0.04( \pm 0.00)$ & $0.06( \pm 0.00)$ \\
\hline AT3G08940 & CP29-2 (LHCB4.2) & PSII & $0.07( \pm 0.03)$ & $0.04( \pm 0.01)$ & $0.04( \pm 0.01)$ & $0.04( \pm 0.00)$ \\
\hline AT1G15820 & CP24 (LHCB6) & PSII & $0.06( \pm 0.02)$ & $0.04( \pm 0.00)$ & $0.04( \pm 0.01)$ & $0.04( \pm 0.00)$ \\
\hline $\begin{array}{l}\text { AT3G27690; AT2G05070; } \\
\text { AT2G05100 }\end{array}$ & LHCB2.4; LHCB2.2; LHCB2.1 & LHCII & $0.04( \pm 0.05)$ & $0.02( \pm 0.00)$ & $0.02( \pm 0.01)$ & $0.01( \pm 0.01)$ \\
\hline ATCG00560 & PSBL & PSII & $0.03( \pm 0.02)$ & $0.02( \pm 0.01)$ & $0.00( \pm 0.00)$ & $0.03( \pm 0.01)$ \\
\hline AT5G66570 & PSBO- $1^{*}$ & PSII & $0.03( \pm 0.01)$ & $0.01( \pm 0.00)$ & $0.01( \pm 0.00)$ & $0.02( \pm 0.00)$ \\
\hline AT5G01530 & CP29-1 (LHCB4.1) & PSII & $0.02( \pm 0.00)$ & $0.02( \pm 0.00)$ & $0.01( \pm 0.01)$ & $0.03( \pm 0.02)$ \\
\hline AT2G34420 & LHCB1.5 & PSII & $0.01( \pm 0.01)$ & $0.01( \pm 0.00)$ & $0.01( \pm 0.00)$ & $0.01( \pm 0.00)^{\mathrm{a}}$ \\
\hline AT5G54270 & LHCB3 & LHCII & $0.01( \pm 0.01)$ & $0.01( \pm 0.00)$ & $0.01( \pm 0.00)$ & $0.01( \pm 0.00)$ \\
\hline
\end{tabular}

Thylakoid membranes were solubilized with digitonin, complexes separated using lpBN-PAGE and selected bands excised for LC-MS/MS analysis. Annotated PSI, PSII and LHC subunits are presented. The quantity of each protein was normalized to PSAA, and the relative protein abundances between genotypes were compared using ANOVA/Brown-Forsythe. Post-hoc analysis of significantly different protein groups (ANOVA $p<0.05)$ was performed using Tukey-HSD. The average relative abundances of detected proteins $( \pm$ standard deviation) are shown $(n=3 ; N=12)$

*ANOVA $p<0.05$

${ }^{\mathrm{a}} n=2$

a direct comparison between different biological replicates or between genotypes, since the raw iBAQ values are not normalized between samples. To overcome this limitation, and to still retain the information of the relative protein abundance within one complex, we normalized the relative protein abundances within each complex to percentage values. Hence, the iBAQ values of all proteins in PSI-containing complexes were normalized to the intensity of the PSI reaction center protein PSAA, to compare the relative abundances of accessory PSI subunits, antenna proteins, and PSII subunits between the genotypes. Proteins detected in the LHCII trimer complexes were normalized to the LHCB3 
Table 2 Quantitative proteome data analysis according to iBAQ values of the proteins identified within the PSI/PSII bands excised from lpBNPAGE gels

\begin{tabular}{|c|c|c|c|c|c|c|}
\hline \multicolumn{2}{|c|}{ Proteins/protein groups } & \multirow[t]{2}{*}{ Subunit of } & \multicolumn{4}{|l|}{ Genotype } \\
\hline Locus/loci & Name(s) & & wt & $n s i-1$ & $n s i-2$ & $\operatorname{stn} 7$ \\
\hline AT4G02770 & PSAD-1 & PSI & $1.73( \pm 0.26)$ & $1.71( \pm 0.13)$ & $1.70( \pm 0.18)$ & $2.08( \pm 0.49)$ \\
\hline AT4G28750 & PSAE-1 & PSI & $1.56( \pm 0.36)$ & $1.37( \pm 0.17)$ & $1.48( \pm 0.26)$ & $1.81( \pm 0.61)$ \\
\hline ATCG01060 & PSAC & PSI & $1.26( \pm 0.05)$ & $1.23( \pm 0.28)$ & $1.08( \pm 0.29)$ & $1.11( \pm 0.19)$ \\
\hline AT1G55670 & PSAG & PSI & $1.21( \pm 0.12)$ & $1.19( \pm 0.15)$ & $1.06( \pm 0.08)$ & $1.16( \pm 0.21)$ \\
\hline ATCG00350 & PSAA & PSI & 1.00 & 1.00 & 1.00 & 1.00 \\
\hline AT3G47470 & LHCA4 & PSI & $0.98( \pm 0.14)$ & $0.85( \pm 0.17)$ & $0.75( \pm 0.07)$ & $1.03( \pm 0.40)$ \\
\hline AT1G61520 & LHCA3 & PSI & $0.97( \pm 0.26)$ & $0.88( \pm 0.29)$ & $0.90( \pm 0.24)$ & $1.10( \pm 0.41)$ \\
\hline AT1G31330 & PSAF & PSI & $0.96( \pm 0.06)$ & $1.10( \pm 0.12)$ & $1.17( \pm 0.25)$ & $0.92( \pm 0.36)$ \\
\hline AT3G54890 & LHCA1 & PSI & $0.70( \pm 0.16)$ & $0.61( \pm 0.14)$ & $0.69( \pm 0.07)$ & $0.67( \pm 0.14)$ \\
\hline ATCG00340 & PSAB & PSI & $0.66( \pm 0.07)$ & $0.62( \pm 0.05)$ & $0.65( \pm 0.04)$ & $0.66( \pm 0.08)$ \\
\hline AT1G52230 & PSAH-2 & PSI & $0.61( \pm 0.19)$ & $0.59( \pm 0.28)$ & $0.69( \pm 0.40)$ & $0.66( \pm 0.45)$ \\
\hline AT3G61470 & LHCA2 & PSI & $0.59( \pm 0.11)$ & $0.45( \pm 0.16)$ & $0.57( \pm 0.16)$ & $0.52( \pm 0.11)$ \\
\hline AT4G12800 & PSAL & PSI & $0.59( \pm 0.13)$ & $0.53( \pm 0.14)$ & $0.53( \pm 0.12)$ & $0.61( \pm 0.16)$ \\
\hline AT1G30380 & PSAK & PSI & $0.41( \pm 0.11)$ & $0.34( \pm 0.05)$ & $0.37( \pm 0.03)$ & $0.61( \pm 0.33)$ \\
\hline AT2G20260 & PSAE-2 & PSI & $0.30( \pm 0.11)$ & $0.22( \pm 0.02)$ & $0.24( \pm 0.05)$ & $0.29( \pm 0.11)$ \\
\hline AT5G64040 & PSAN & PSI & $0.08( \pm 0.04)$ & $0.09( \pm 0.05)$ & $0.10( \pm 0.05)$ & $0.10( \pm 0.05)$ \\
\hline AT1G08380 & PSAO & PSI & $0.02( \pm 0.01)$ & $0.01( \pm 0.00)$ & $0.03( \pm 0.02)$ & $0.02( \pm 0.01)$ \\
\hline ATCG00680 & CP47 (PSBB) & PSII & $0.52( \pm 0.00)$ & $0.47( \pm 0.00)$ & $0.45( \pm 0.00)$ & $0.62( \pm 0.00)$ \\
\hline ATCG00280 & CP43 (PSBC)* & PSII & $0.49( \pm 0.00)$ & $0.39( \pm 0.00)$ & $0.34( \pm 0.00)$ & $0.53( \pm 0.00)$ \\
\hline ATCG00270 & D2 (PSBD) & PSII & $0.37( \pm 0.00)$ & $0.28( \pm 0.00)$ & $0.26( \pm 0.00)$ & $0.29( \pm 0.00)$ \\
\hline ATCG00020 & D1 (PSBA) & PSII & $0.27( \pm 0.00)$ & $0.23( \pm 0.00)$ & $0.20( \pm 0.00)$ & $0.26( \pm 0.00)$ \\
\hline AT5G66570 & PSBO-1 & PSII & $0.12( \pm 0.00)$ & $0.10( \pm 0.00)$ & $0.10( \pm 0.00)$ & $0.15( \pm 0.00)$ \\
\hline $\begin{array}{l}\text { AT1G29930; } \\
\text { AT1G29920; } \\
\text { AT1G29910 }\end{array}$ & $\begin{array}{l}\text { LHCB1.3; } \\
\text { LHCB1.1; } \\
\text { LHCB1.2 }\end{array}$ & LHCII & $0.09( \pm 0.00)$ & $0.08( \pm 0.00)$ & $0.08( \pm 0.00)$ & $0.09( \pm 0.00)$ \\
\hline AT4G10340 & CP26 (LHCB5) & PSII & $0.07( \pm 0.00)$ & $0.06( \pm 0.00)$ & $0.05( \pm 0.00)$ & $0.08( \pm 0.00)$ \\
\hline ATCG00560 & PSBL & PSII & $0.06( \pm 0.00)^{\mathrm{a}}$ & $0.02( \pm 0.00)^{\mathrm{a}}$ & $0.02( \pm 0.00)$ & $0.08( \pm 0.00)^{\mathrm{a}}$ \\
\hline AT3G08940 & CP29.2 (LHCB4.2) & PSII & $0.01( \pm 0.00)$ & $0.01( \pm 0.00)$ & $0.01( \pm 0.00)$ & $0.04( \pm 0.00)$ \\
\hline AT3G50820 & PSBO- $2^{*}$ & PSII & $0.01( \pm 0.00)$ & $0.01( \pm 0.00)$ & $0.01( \pm 0.00)$ & $0.02( \pm 0.00)$ \\
\hline AT2G05070 & LHCB 2.2 & LHCII & $0.03( \pm 0.00)$ & $0.02( \pm 0.00)$ & $0.01( \pm 0.00)$ & $0.02( \pm 0.00)$ \\
\hline AT2G34430 & LHCB1.4 & LHCII & $0.02( \pm 0.01)$ & $0.01( \pm 0.01)$ & $0.01( \pm 0.00)$ & $0.01( \pm 0.01)$ \\
\hline
\end{tabular}

Thylakoid membranes were solubilized with dodecyl maltoside, complexes separated using lpBN-PAGE and selected bands excised for LC-MS/ MS analysis. Annotated PSI, PSII and LHC subunits are presented. The quantity of each protein was normalized to PSAA, and the relative protein abundances between genotypes were compared using ANOVA/Brown-Forsythe. Post-hoc analysis of significantly different protein groups (ANOVA $p<0.05)$ was performed using Tukey-HSD. The average relative abundances of detected proteins $( \pm$ standard deviation) are shown $(n=3 ; N=12)$

*ANOVA $p<0.05$

${ }^{\mathrm{a}} n=2$

subunit of PSII antenna, since this subunit is not present in the mobile L-LHCII antenna (Galka et al. 2012), and therefore, allows us to estimate whether there is a change in the distribution of different types of antenna trimers found in particular thylakoid domains (i.e. non-appressed thylakoids and whole thylakoids) between the genotypes. Only proteins whose abundance was more than $1.0 \%$ of PSAA/ LHCB3 were included in the analysis, since very low abundance proteins were considered likely contaminants due to imperfect separation during lpBN gel electrophoresis. Additionally, only proteins with MS-score $>40$ were considered reliably detected and included in the analysis. Normalized iBAQ values for each complex (digitonin-solubilized PSI complex and LHCII trimer, and DM-solubilized PSI/PSII dimer complex and LHCII trimer, Fig. 2) were used for statistical analyses with ANOVA in SPSS statistics software (IBM) and differences in the normalized relative abundances between genotypes were tested. If variances between 
Table 3 Quantitative proteome data analysis according to iBAQ values of the proteins identified within the LHCII trimer bands excised from lpBN-PAGE gels

\begin{tabular}{|c|c|c|c|c|c|c|}
\hline \multicolumn{2}{|c|}{ Proteins/protein groups } & \multirow[t]{2}{*}{ Subunit of } & \multicolumn{4}{|l|}{ Genotype } \\
\hline Locus/loci & Name(s) & & wt & $n s i-1$ & $n s i-2$ & $\sin 7$ \\
\hline AT2G05070 & LHCB2.2 & LHCII & $8.29( \pm 0.75)$ & $15.21( \pm 10.94)$ & $14.34( \pm 6.24)$ & $11.25( \pm 1.84)$ \\
\hline AT2G34420 & LHCB 1.5 & LHCII & $3.08( \pm 0.96)$ & $2.34( \pm 0.53)$ & $4.17( \pm 1.59)$ & $3.27( \pm 1.37)$ \\
\hline AT5G54270 & LHCB3 & LHCII & 1.00 & 1.00 & 1.00 & 1.00 \\
\hline AT2G34430 & LHCB 1.4 & LHCII & $0.43( \pm 0.12)$ & $0.58( \pm 0.17)$ & $0.82( \pm 0.06)$ & $0.78( \pm 0.33)$ \\
\hline ATCG00680 & CP47 (PSBB) & PSII & $0.29( \pm 0.05)$ & $0.58( \pm 0.27)$ & $0.65( \pm 0.13)$ & $0.46( \pm 0.08)$ \\
\hline ATCG00020 & D1 (PSBA) & PSII & $0.28( \pm 0.08)$ & $0.51( \pm 0.27)$ & $0.63( \pm 0.14)$ & $0.52( \pm 0.10)$ \\
\hline ATCG00270 & D2 (PSBD) & PSII & $0.26( \pm 0.04)$ & $0.65( \pm 0.28)$ & $0.75( \pm 0.20)$ & $0.58( \pm 0.11)$ \\
\hline AT1G44575 & NPQ4 (PSBS) & LHCII & $0.23( \pm 0.06)$ & $0.38( \pm 0.07)$ & $0.36( \pm 0.02)$ & $0.33( \pm 0.12)$ \\
\hline AT1G34000 & $\mathrm{OHP} 2$ & aux & $0.18( \pm 0.01)$ & $0.41( \pm 0.13)$ & $0.46( \pm 0.17)$ & $0.36( \pm 0.10)$ \\
\hline AT4G10340 & CP26 (LHCB5) & PSII & $0.16( \pm 0.05)$ & $0.25( \pm 0.05)$ & $0.22( \pm 0.01)$ & $0.20( \pm 0.06)$ \\
\hline AT5G64040 & PSAN & PSI & $0.16( \pm 0.08)$ & $0.40( \pm 0.14)$ & $0.50( \pm 0.07)$ & $0.42( \pm 0.20)$ \\
\hline AT5G02120 & OHP1* & aux & $0.14( \pm 0.04)$ & $0.26( \pm 0.04)$ & $0.29( \pm 0.07)$ & $0.23( \pm 0.03)$ \\
\hline AT1G29930 & LHCB 1.3 & LHCII & $0.11( \pm 0.05)$ & $0.08( \pm 0.00)$ & $0.18( \pm 0.18)$ & $0.27( \pm 0.17)$ \\
\hline AT3G47470 & LHCA4 & PSI & $0.11( \pm 0.02)$ & $0.36( \pm 0.39)$ & $0.23( \pm 0.11)$ & $0.20( \pm 0.01)$ \\
\hline ATCG00280 & CP43 (PSBC)* & PSII & $0.07( \pm 0.01)$ & $0.15( \pm 0.04)$ & $0.17( \pm 0.02)$ & $0.13( \pm 0.03)$ \\
\hline AT3G54890 & LHCA1 & PSI & $0.07( \pm 0.01)$ & $0.18( \pm 0.14)$ & $0.12( \pm 0.02)$ & $0.11( \pm 0.02)$ \\
\hline AT1G31330 & PSAF & PSI & $0.07( \pm 0.04)$ & $0.20( \pm 0.17)$ & $0.15( \pm 0.05)$ & $0.17( \pm 0.06)$ \\
\hline AT1G61520 & LHCA3 & PSI & $0.07( \pm 0.02)$ & $0.20( \pm 0.10)$ & $0.15( \pm 0.05)$ & $0.11( \pm 0.02)$ \\
\hline AT1G52230 & PSAH-2 & PSI & $0.06( \pm 0.04)$ & $0.38( \pm 0.49)$ & $0.22( \pm 0.15)$ & $0.15( \pm 0.13)$ \\
\hline AT2G46820 & PSAP & PSI & $0.06( \pm 0.01)$ & $0.12( \pm 0.00)$ & $0.18( \pm 0.06)$ & $0.12( \pm 0.03)$ \\
\hline AT1G79040 & PSAR & PSI & $0.05( \pm 0.00)$ & $0.07( \pm 0.02)$ & $0.08( \pm 0.01)$ & $0.08( \pm 0.03)$ \\
\hline AT3G08940 & CP29-2 (LHCB4.2) & PSII & $0.05( \pm 0.01)$ & $0.08( \pm 0.02)$ & $0.07( \pm 0.01)$ & $0.06( \pm 0.01)$ \\
\hline AT5G66570 & PSBO-1 & PSII & $0.05( \pm 0.02)$ & $0.08( \pm 0.03)$ & $0.08( \pm 0.03)$ & $0.09( \pm 0.06)$ \\
\hline $\begin{array}{l}\text { AT3G61470; } \\
\text { AT5G28450 }\end{array}$ & LHCA2; CAB family & PSI & $0.04( \pm 0.01)$ & $0.10( \pm 0.08)$ & $0.08( \pm 0.02)$ & $0.06( \pm 0.02)$ \\
\hline ATCG00340 & PSAB & PSI & $0.04( \pm 0.01)$ & $0.16( \pm 0.14)$ & $0.12( \pm 0.03)$ & $0.09( \pm 0.04)$ \\
\hline AT1G15820 & CP24 (LHCB6) & PSII & $0.03( \pm 0.01)$ & $0.04( \pm 0.01)$ & $0.04( \pm 0.01)$ & $0.04( \pm 0.01)$ \\
\hline AT4G28750 & PSAE-1 & PSI & $0.03( \pm 0.02)$ & $0.10( \pm 0.06)$ & $0.08( \pm 0.01)$ & $0.07( \pm 0.04)$ \\
\hline ATCG00350 & PSAA & PSI & $0.03( \pm 0.00)$ & $0.10( \pm 0.07)$ & $0.09( \pm 0.02)$ & $0.07( \pm 0.01)$ \\
\hline AT5G51545 & LPA2 & aux & $0.03( \pm 0.02)$ & $0.04( \pm 0.02)$ & $0.07( \pm 0.02)$ & $0.06( \pm 0.03)$ \\
\hline AT5G01530 & CP29-1 (LHCB4.1) & PSII & $0.02( \pm 0.01)$ & $0.03( \pm 0.00)$ & $0.03( \pm 0.01)$ & $0.03( \pm 0.01)$ \\
\hline AT4G12800 & PSAL & PSI & $0.02( \pm 0.01)$ & $0.07( \pm 0.07)$ & $0.04( \pm 0.02)$ & $0.03( \pm 0.02)$ \\
\hline AT4G02770 & PSAD-1 & PSI & $0.01( \pm 0.00)$ & $0.07( \pm 0.07)$ & $0.05( \pm 0.02)$ & $0.03( \pm 0.02)$ \\
\hline AT1G03600 & PSB27 & aux & $0.01( \pm 0.00)$ & $0.03( \pm 0.01)$ & $0.03( \pm 0.01)$ & $0.04( \pm 0.02)$ \\
\hline ATCG00560 & PSBL & PSII & $0.01( \pm 0.01)$ & $0.02( \pm 0.00)$ & $0.02( \pm 0.00)$ & $0.02( \pm 0.02)^{\mathrm{a}}$ \\
\hline AT1G08380 & PSAO & PSI & $0.01^{\mathrm{b}}$ & $0.07( \pm 0.09)^{\mathrm{a}}$ & $0.04^{\mathrm{b}}$ & $0.01( \pm 0.00)^{\mathrm{a}}$ \\
\hline AT1G30380 & PSAK & PSI & $0.01( \pm 0.00)$ & $0.04( \pm 0.05)$ & $0.01( \pm 0.01)$ & $0.01( \pm 0.00)$ \\
\hline
\end{tabular}

Thylakoid membranes were solubilized with digitonin, complexes separated using lpBN-PAGE and selected bands excised for LC-MS/MS analysis. Annotated PSI, PSII and LHC subunits as well as auxiliary (aux.) PS components are presented. The quantity of each protein was normalized to LHCB3, and the relative protein abundances between genotypes were compared using ANOVA/Brown-Forsythe. Post-hoc analysis of significantly different protein groups (ANOVA $p<0.05$ ) was performed using Tukey-HSD. The average relative abundances of detected proteins ( \pm standard deviation) are shown $(n=3 ; N=12$ ). The spectrum file was re-analyzed from Koskela et al. (2018) (www.plantcell.org, copyright American Society of Plant Biologists)

*ANOVA $p<0.05$

${ }^{\mathrm{a}} n=2$

${ }^{\mathrm{b}} n=1$ 
genotypes were significantly different (Levene test $p<0.05$ ), the robust Brown-Forsythe test was used instead. If a protein was detected in only one replicate for a genotype, that genotype was omitted from statistical analysis for that protein. Missing values (i.e. cases where protein was not detected in a replicate) were set as 'system missing'. Because peptide/ protein abundances were found to be significantly different (ANOVA or Brown-Forsythe $p<0.05$ ) only in cases where variances between groups were homogenous, post hoc analysis was performed using Tukey-HSD. $p$ values for statistical analyses are shown in Supplemental Tables 3-7.

\section{Results}

\section{stn7 and nsi mutants under fluctuating light conditions}

The $\sin 7$ knock-out plants are deficient in the short-term regulation of the photosynthetic light reactions via LHCII phosphorylation (Depège et al. 2003; Bellafiore et al. 2005). Interestingly, the $\sin 7$ mutant does not show growth defects under constant light conditions, but exposure to fluctuating light causes a dramatic retardation in growth (Bellafiore et al. 2005; Tikkanen et al. 2010). The nsi loss-of-function lines are not compromised in the phosphorylation of thylakoid proteins, but they are incapable of performing state transitions (Koskela et al. 2018). Nevertheless, the phenotype of the nsi plants did not markedly differ from wt controls under standard growth conditions (Koskela et al. 2018). To find out whether the retarded growth of $\sin 7$ under fluctuating light is due to defects in state transitions alone, 2-week old wt, $n s i-1, n s i-2$, and $\operatorname{stn} 7$ plants were treated for 2 weeks with fluctuating light (cycles of 4 min $90 \mu \mathrm{mol}$ photons $\mathrm{m}^{-2} \mathrm{~s}^{-1}$ followed by a $1 \mathrm{~min} 900 \mu \mathrm{mol}$ photons $\mathrm{m}^{-2} \mathrm{~s}^{-1}$ ). Indeed, $\operatorname{stn} 7$ as well as both $n s i$ lines ( $n s i-1$ and $n s i-2)$ showed significantly reduced growth under fluctuating light conditions (Fig. 1a, b). The fluctuating light treatment also decreased the chlorophyll content of all plant lines (Fig. 1c). In wt, fluctuating light increased the chlorophyll $\mathrm{a} / \mathrm{b}$ ratio, while in all mutant lines the chlorophyll $\mathrm{a} / \mathrm{b}$ ratio slightly decreased (Fig. 1d).

To gain insights into the photosynthetic properties of the $n s i$ and $\sin 7$ plants, maximum PSII quantum yield $\left(F_{\mathrm{V}} / F_{\mathrm{M}}\right)$ of the plants grown under standard growth conditions or treated with fluctuating light (cycles of $4 \mathrm{~min} 90 \mu \mathrm{mol}$ photons $\mathrm{m}^{-2} \mathrm{~s}^{-1}$ followed by a $1 \mathrm{~min} 900 \mu \mathrm{mol}$ photons $\mathrm{m}^{-2} \mathrm{~s}^{-1}$ ) were determined using an IMAGING-PAM fluorometer. $F_{\mathrm{V}} / F_{\mathrm{M}}$ in wt plants decreased sharply within the two first days of fluctuating light treatment as compared to the plants grown under standard conditions. However, during the 2-week treatment, the PSII capacity recovered almost to the same level as detected under standard growth conditions
(Fig. 1e). In contrast, $F_{\mathrm{V}} / F_{\mathrm{M}}$ in the three loss-of-function mutant lines revealed a uniform and drastic decrease, which did not recover throughout the 2-week fluctuating light treatment (Fig. 1e). In line with maximum PSII efficiency ( $\mathrm{F}_{\mathrm{V}} /$ $\mathrm{F}_{\mathrm{M}}$ ), fluctuating light did not affect the PSII yield (YII) of wt plants (Fig. 1f). In all mutant lines, however, the PSII yield was drastically decreased by the fluctuating light treatment (Fig. 1f). In both $\sin 7$ and wt, the level of steady-state NPQ was not dramatically affected by fluctuating light treatment, as compared to constant light, even if in $\operatorname{stn} 7 \mathrm{NPQ}$ was consistently lower than in wt (Fig. 1g). In both $n s i$ lines, NPQ levels were even lower than in $\sin 7$ even under standard growth conditions. Intriguingly, fluctuating light treatment of the plants resulted in a drastic decrease in NPQ only in the nsi mutants (Fig. 1g). Moreover, fluctuating light treatment resulted in an increase of quantum yield of non-regulated energy dissipation $\mathrm{Y}(\mathrm{NO})$, expressed as the average of the last two data points on the induction curve on day 15 $(n=6)$, especially in the $n s i$ mutant plants: for $n s i-1, \mathrm{Y}(\mathrm{NO})$ increased from $0.31 \pm 0.02$ under standard light conditions to $0.55 \pm 0.02$ under fluctuating light, and for $n s i-2$ the increase was from $0.31 \pm 0.02$ to $0.54 \pm 0.02$. In $\operatorname{stn} 7$, the increase was smaller (from $0.27 \pm 0.02$ to $0.41 \pm 0.01$ ) than in $n s i$. In contrast to the mutants, $\mathrm{Y}(\mathrm{NO})$ in wt remained constant $(0.28 \pm 0.02)$ under standard conditions and under fluctuating light $(0.29 \pm 0.02)$.

\section{Composition of thylakoid protein complexes}

To define the structural differences of the main players of state transitions, i.e. PSI and LHCII, between wt, $\sin 7$ and nsi mutants, the plants were grown under standard conditions for 5 weeks, and thylakoids were isolated in the middle of the light period, where wild type plants are in state 2 (Suorsa et al. 2015). Thereafter, thylakoids were solubilized with commonly used detergents digitonin or $\beta$-DM (hereafter DM). Digitonin has been used to study weak protein-protein interactions in the non-appressed regions of thylakoids, while DM solubilizes the entire thylakoid membrane, but is unable to maintain labile interactions between the protein complexes (Järvi et al. 2011). Indeed, the chlorophyll $\mathrm{a} / \mathrm{b}$ ratio of the digitonin-solubilized wt thylakoid fraction was $4.34 \pm 0.244$ and that of the DM-solubilized thylakoids $3.21 \pm 0.033(n=3)$. As the chlorophyll a/b ratio of total thylakoids was $3.20 \pm 0.022$, it is clear that DM solubilizes the entire thylakoid membrane, while digitonin solubilizes less $\mathrm{Chl}$ b-containing grana membranes (i.e. non-appressed thylakoid fraction). These values are in accordance with the previously published results (e.g. Kyle et al. 1983; Rantala et al. 2017; Koochak et al. 2019; Trotta et al. 2019) and also with the results obtained by mechanical fractionation (e.g. Danielsson et al. 2006; Suorsa et al. 2014). Thereafter, solubilized thylakoid protein complexes were separated 


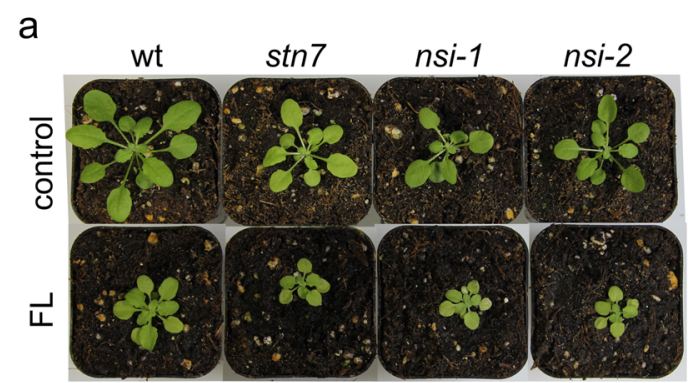

C

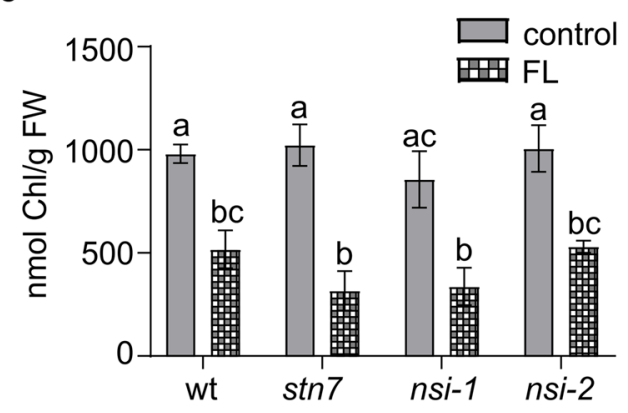

e

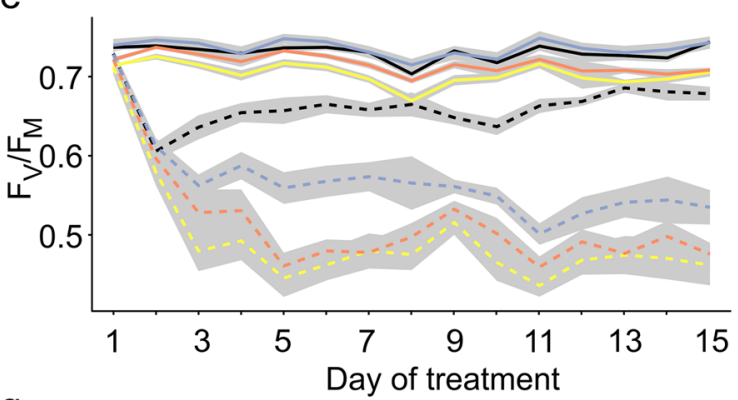

9

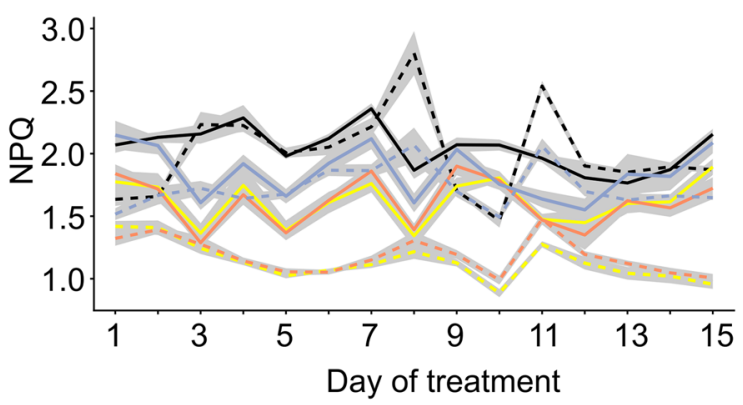

b
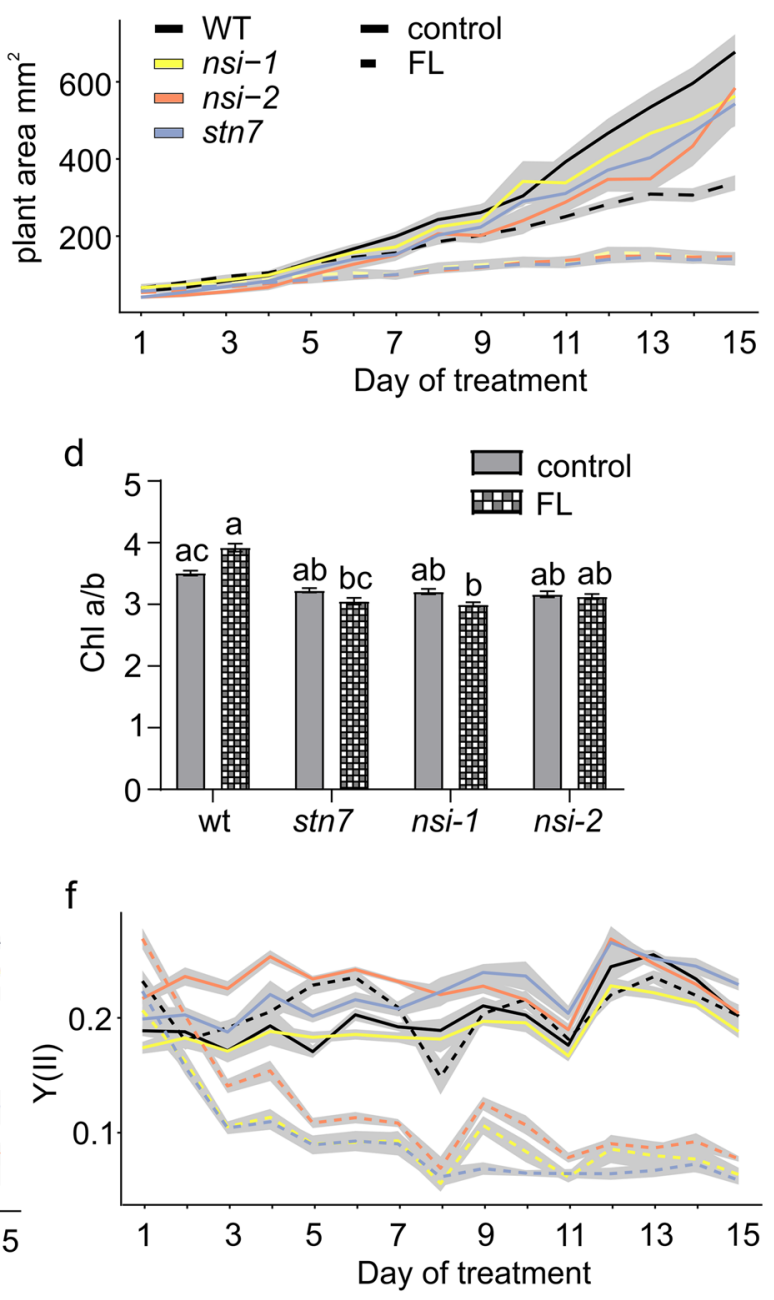

Fig. 1 Growth and photosynthetic properties of Arabidopsis thaliana wild type (wt), stn 7 and $n s i$ plants grown under standard and fluctuating light conditions. a Visual phenotype, b area, $\mathbf{c}$ chlorophyll (Chl) $\mathrm{a}$ and $\mathrm{b}$ content, $\mathbf{d} \mathrm{Chl} \mathrm{a} / \mathrm{b}$ ratio, e maximum quantum efficiency of PSII photochemistry (monitored as $F_{\mathrm{V}} / F_{\mathrm{M}}$ ), f steady-state PSII yield (Y(II)), $\mathbf{g}$ non-photochemical quenching (NPQ/4) of the plants grown under standard conditions for 4 weeks (control) or for 2 weeks under standard conditions followed by 2 weeks growth under fluctuating light (FL; 4 min of $90 \mu \mathrm{mol}$ photons $\mathrm{m}^{-2} \mathrm{~s}^{-1}$ and $1 \mathrm{~min}$ of $900 \mu \mathrm{mol}$ photons $\mathrm{m}^{-2} \mathrm{~s}^{-1}$ ). a, $\mathbf{c}, \mathbf{d}$ represent values from mature plants and $\mathbf{b}$, e, f, $\mathbf{g}$ show results from daily measurements during the 2 weeks FL treatment. Black line in $\mathbf{b}, \mathbf{e}, \mathbf{f}$ and $\mathbf{g}$ denotes wt, blue $\sin 7$, yellow $n s i$ 1 and orange $n s i-2$. Solid lines represent plants grown under standard light conditions and dotted lines FL treatment. $\mathbf{f}$, $\mathbf{g}$ shows average of last two data points on the induction curve. Shaded region in $\mathbf{b}, \mathbf{e}, \mathbf{f}$, g represents $+/-1$ standard error. Six plants were used per genotype and per treatment (4) 


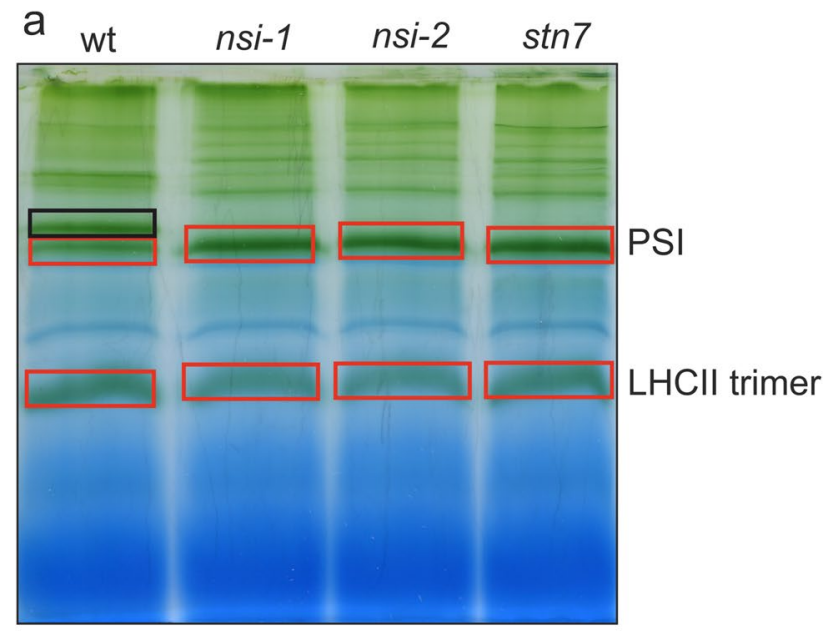

b

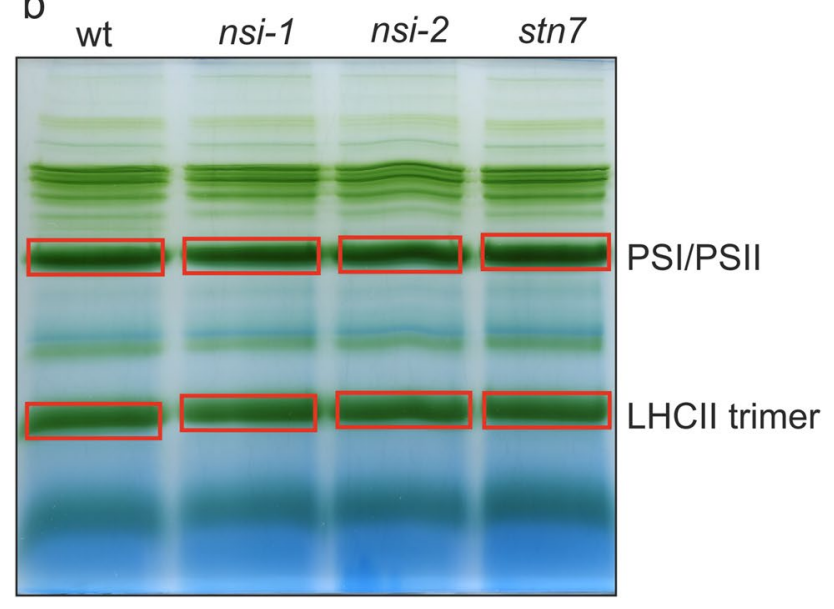

Fig. 2 Thylakoid protein complexes of wt, nsi-1, nsi-2, and $\operatorname{stn} 7$ plants. Plants were grown under standard conditions $(100 \mu \mathrm{mol}$ photons $\mathrm{m}^{-2} \mathrm{~s}^{-1}, 8 \mathrm{~h} / 16 \mathrm{~h}$ light/dark), thylakoids solubilized either with a $1 \%(\mathrm{w} / \mathrm{v})$ digitonin (solubilization of grana margins and stroma lamellae) or b $1 \%(\mathrm{w} / \mathrm{v})$ dodecyl maltoside (solubilization of the entire thylakoid network), and protein complexes separated using lpBN gel electrophoresis. $50 \mu \mathrm{g}$ of chlorophyll was loaded in (a) and $30 \mu \mathrm{g}$ in (b). Slices indicated by red boxes were cut from the gels and subjected to LC-MS/MS. The black box indicates the PSI-LHCII complex (state transition complex) present in the grana margins or stroma lamellae of wt plants

using $\mathrm{lpBN}$ gel electrophoresis, and selected protein complexes were analyzed by LC-MS/MS (Fig. 2). As previously shown, the overall accumulation of the thylakoid protein complexes was similar in all plant lines, and no visual differences could be detected when thylakoids were solubilized with DM (Fig. 2b; Tikkanen et al. 2008; Koskela et al. 2018). Nevertheless, the well-characterized state transition complex composed of PSI, LHCI and LHCII was missing in $\operatorname{stn} 7$ and $n s i$ mutants, when thylakoids were solubilized with digitonin, and also the megacomplex composition of the mutants differed from that of the wt (Fig. 2a; Pesaresi et al. 2009; Suorsa et al 2015; Koskela et al. 2018). We also took a closer look at the thylakoid protein complexes of $n s i$ by running a $2 \mathrm{D}-\mathrm{BN}$ gel, but apart from the lack of PSI-LHCII complex, no major differences in the subunit composition of $n s i-1$ or $n s i-2$ thylakoid complexes could be detected as compared to wt (Fig. S1, Fig. 3).

\section{PSI/PSII band}

The PSI/PSII band of the non-appressed thylakoids, obtained by digitonin solubilization and lpBN gel electrophoresis (defined in Fig. 2a), was analyzed by LC-MS/MS. This band has previously shown to contain mainly PSI subunits (Suorsa et al. 2015), which was also the major constituent of the band in our iBAQ analysis (Table 1). Comparison of wt and $\sin 7$ and $n s i$ mutant lines showed equal accumulation of the PSI core subunit PSAB, PSI proteins facing the lumen (PSAN and PSAF) as well as the PSI proteins forming the stromal ridge (PSAC, PSAD and PSAE) responsible for ferredoxin recruitment (Table 1, Supplemental Table S1). Neither the relative abundance of the proteins involved in the binding of LHCII to PSI (PSAH, PSAL and PSAO) (Lunde et al. 2000; Schöttler et al. 2011; Pan et al. 2018) nor the minor PSI proteins (PSAG, PSAK, PSAP) differed between the mutant lines as compared to wt. Moreover, no differences were detected in the accumulation of LHCA1-4 proteins (Table 1). Small amounts of PSII core dimers have been shown to co-migrate in this band together with PSI complexes (Suorsa et al. 2015; Rantala et al. 2017). Our analysis confirmed that PSII proteins were less abundant (only up to $25 \%$ ) in this band as compared to PSAA. PSII core proteins D1 and D2, inner antenna proteins PSBB and PSBC, as well as the minor PSII protein PSBL accumulated in equal quantities in all plant lines (Table 1). It should be noted, however, that the low abundance of PSII proteins does not allow reliable estimation of PSI/PSII ratio within this band and would not be relevant since the majority of PSII complexes are left insolubilized during digitonin solubilization and are thus not involved in the analysis. Oxygen-evolving complex proteins PSBO-1, PSBO-2 and PSBR were also represented within the samples. Interestingly, the amount of PSBO-2 in the PSII dimer migrating in the PSI/PSII band was decreased to around $60 \%$ in the nsi mutants as compared to wt ( $p=0.004$ and 0.011 for $n s i-2$ and $n s i-1$, respectively). In $\operatorname{stn} 7$ the accumulation of PSBO-2 was also slightly decreased compared to wt (around 85\% of wt level), but the difference was not significant ( $p=0.374)$, while a significant difference remained between $n s i-2$ and $\operatorname{stn} 7(p=0.043)$. Similarly, although less pronounced, $n s i-l$ had less PSBO- 2 than $\sin 7(p=0.114)$. The PSBO-1 isoform also showed lower accumulation in the nsi mutants as compared to wt, although with less significance compared to PSBO-2 ( $p$ values $=0.054$ and 0.075 for $n s i-1$ and $n s i-2$, respectively). Although mainly photosystem core complexes are known to migrate in this 
Fig. 3 2D-Blue Native gels of wt and nsi-2 thylakoid protein complexes. Thylakoid samples from growth light $(100 \mu \mathrm{mol}$ photons $\left.\mathrm{m}^{-2} \mathrm{~s}^{-1}\right)$ adapted plants were solubilized either with a $1 \%(\mathrm{w} / \mathrm{v})$ digitonin $(5 \mu \mathrm{g}$ of chlorophyll) or b $1 \%(\mathrm{w} / \mathrm{v})$ dodecyl maltoside $(3.5 \mu \mathrm{g}$ of chlorophyll) prior to lpBN gel electrophoresis. lpBN gel electrophoresis was followed by separation of protein complexes in the second dimension on $12 \%$ reducing SDS-PAGE supplemented with $6 \mathrm{M}$ urea and SYPRO staining. The proteins were identified based on Aro et al. 2005 and Suorsa et al. 2015. sc, supercomplex; PSIId, PSII dimer; PSIIm, PSII monomer; Cytb $_{6} \mathrm{f}$, cytochromes $\mathrm{b}_{6} \mathrm{f}$

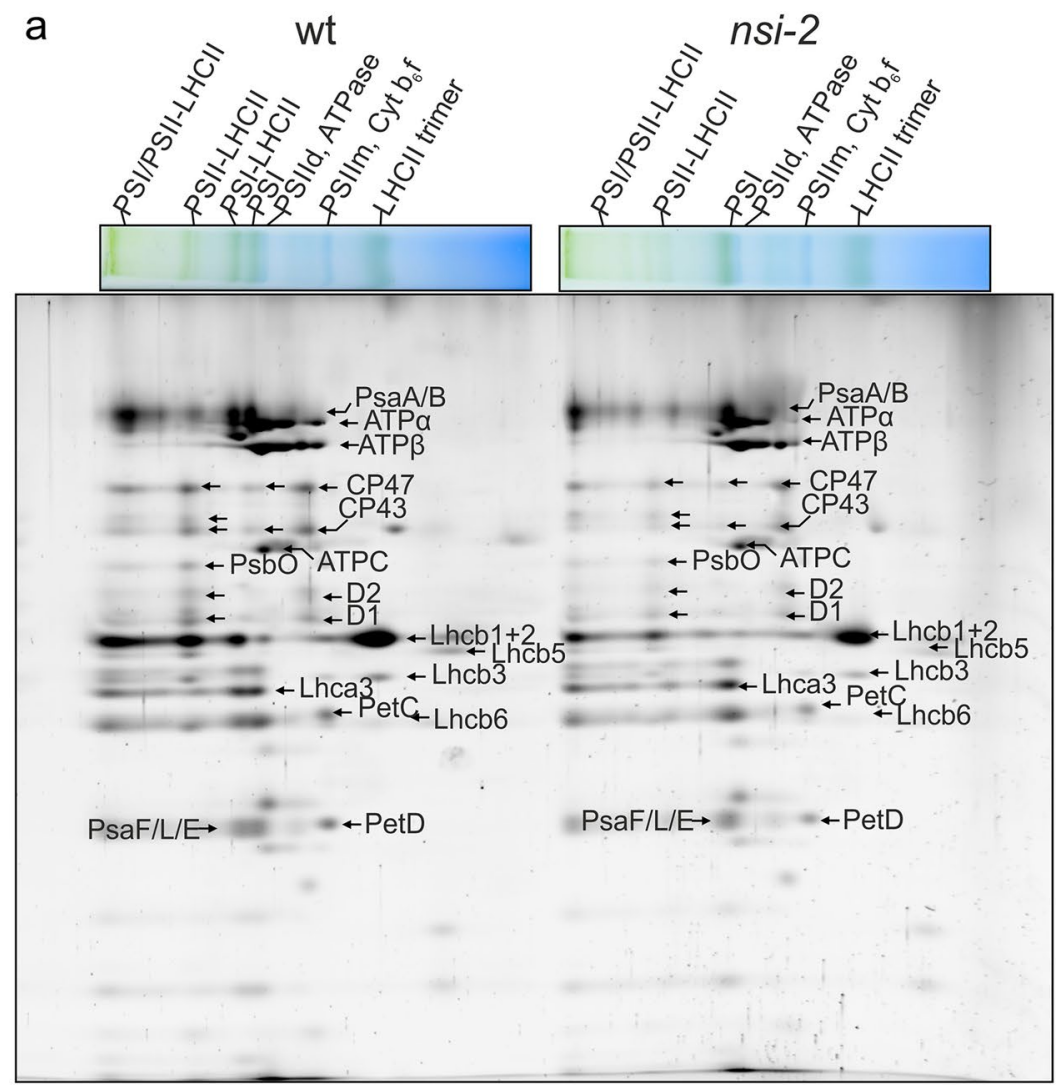

b wt

$n s i-2$

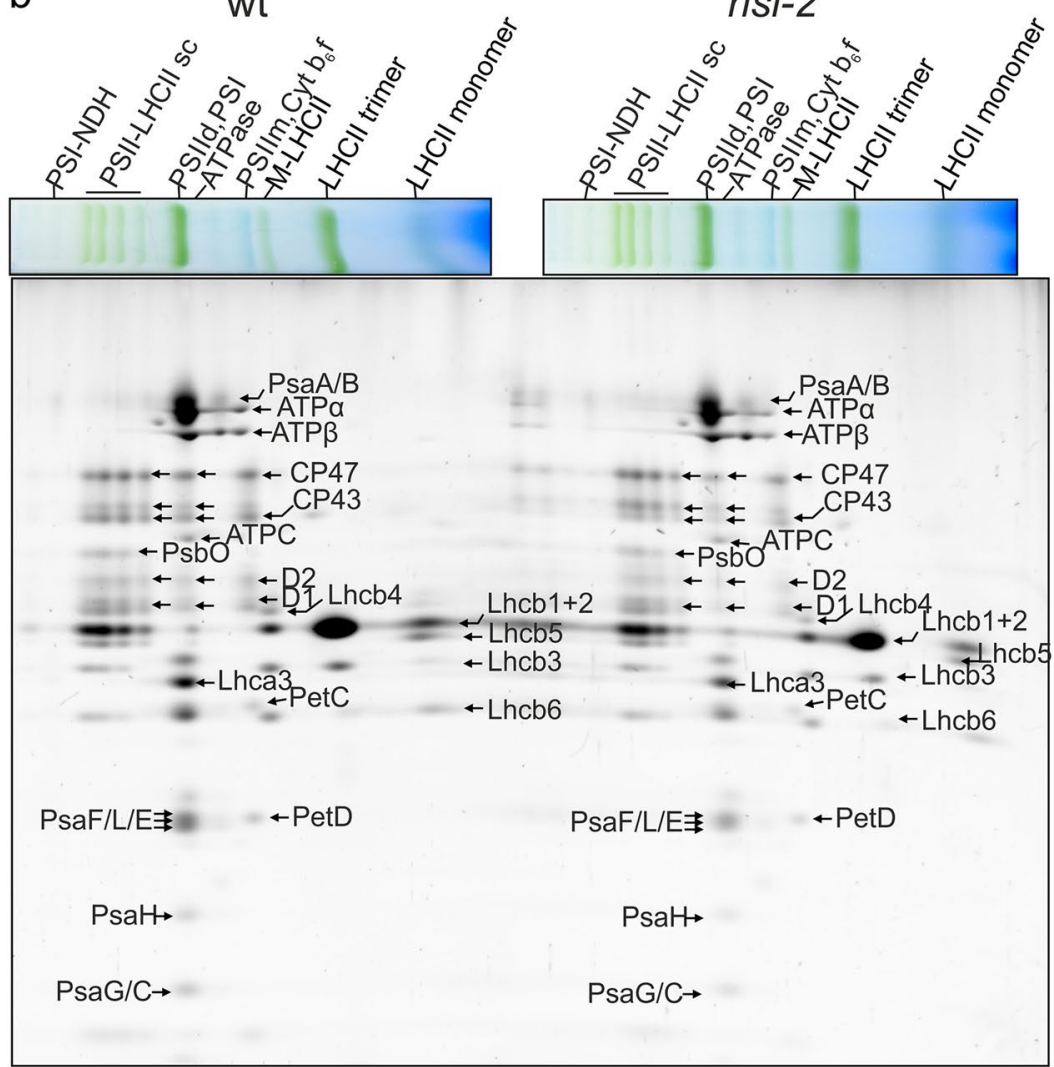


band, also several peptides representing different isoforms of LHCB1 (LHCB1.1/1.2, LHCB1.5), LHCB2 (LHCB2.1/2/4) and LHCB4 (LHCB4.1, LHCB4.2, LHCB4.3) as well as LHCB3, LHCB5 and LHCB6 proteins were detected in all plant lines. Significantly less peptides, around $40 \%$ of wt level, representing LHCB1.1 and LHCB1.2 were observed in the PSI/PSII band of digitonin-solubilized $\sin 7$ thylakoids as compared to wt (Table 1) $(p=0.019)$. In nsi mutants, the amount of LHCB1.1/1.2 in the band was also reduced to $60 \%$ in $n s i-1(p=0.142)$ and $50 \%$ in $n s i-2(p=0.019)$ when compared with wt, while there was no significant difference between $n s i$ and $\operatorname{stn} 7$ mutants. However, as the protein complex pattern in wt is very different from that of the mutants (which lack the PSI-LHCII complex), direct comparison of the LHCII protein abundance to PSI is not possible.

The PSI/PSII band was also analyzed from thylakoid samples solubilized with DM (band defined in Fig. 2b), and it was shown to contain a similar complement of proteins as did the PSI/PSII band from thylakoids solubilized with digitonin (Table 2). The most abundant PSI proteins (normalized to PSAA) were PSAD, PSAE, PSAC, and PSAG (Table 2). In addition, many other PSI subunits as well as LHCA1-4 proteins were detected. Since DM-solubilized thylakoids contain also the appressed grana thylakoids, which host the majority of the PSII complexes, the PSII proteins were more abundant in the PSI/PSII band of DM-solubilized samples as compared to digitonin-solubilized samples (Table 2), reaching up to half of the PSAA amount. This result is in line with earlier studies showing enrichment of PSI subunits in digitonin-solubilized thylakoids (Rantala et al. 2017; Koochak et al. 2019; Trotta et al. 2019). Interestingly, the CP43/PSAA ratio in the PSI/PSII band of the DM-solubilized thylakoids was $30 \%$ lower in $n s i$ as compared to $\operatorname{stn} 7$ ( $p=0.023$ and 0.085 for $n s i-2$ and $n s i-1$, respectively). In fact, a similar trend was observed for all PSII core subunit (D1, D2, CP43 and CP47): their amount was slightly reduced in $n s i$ as compared to $\operatorname{stn} 7$ and wt, although the differences were not statistically significant apart from the above-mentioned CP43. Moreover, 2D gel electrophoresis analysis revealed that the amount of phospho-CP43 (upper band) seemed higher and the amount of non-phosphorylated CP43 (lower band) lower in $n s i-2$ as compared to wt (Fig. 3).

Similar to the non-appressed thylakoids, analysis of the PSI/PSII band of DM-solubilized thylakoids showed that $n s i$ mutants contained less PSBO-2 than wt and $\operatorname{stn} 7$. However, in DM-solubilized thylakoids, the difference between $n s i$ and wt was less pronounced (PSBO-2 level of $n s i$ around $85 \%$ of $w t)$ and statistically non-significant, whereas the amount in $\sin 7$ was increased compared to wt $(p=0.095)$. Overall, the only statistically significant difference in PSBO-2 amount in whole thylakoids was between both $n s i$ mutants and $\sin 7$ ( $p=0.044$ and 0.048 for $n s i-2$ and $n s i-1$, respectively), with $n s i-1$ and $n s i-2$ having around $40 \%$ less PSBO-2 in this complex.

\section{LHCII trimers}

During lpBN gel electrophoresis, large amount of LHCII trimers (Fig. 2) disconnect from the photosystems due to solubilization (Tikkanen et al. 2008, 2012). Previously, it has been shown that LHCB1 and LHCB2 are the most abundant proteins in the disconnected "free" LHCII trimers (Galka et al. 2012). Accordingly, Table 3 shows that LHCII trimers in digitonin-solubilized thylakoids possessed high quantities of LHCB2.2 and LHCB1.5 as normalized to LHCB3, which, in line with previous findings (Galka et al. 2012; Rantala et al. 2017), was ten times less abundant than LHCB2.2. In addition to LHCB2.2, LHCB1.5 and LHCB3, the trimers contained LHCB1.4 and traces of LHCB1.3, LHCB5 and LHCB4.1-4.3. In addition, a number of PSII and PSI proteins as well as LHCA1-LHCA4 were detected, and the amount of CP43 was higher in both nsi mutants as compared to wt and $\sin 7$. Additionally, in all plant lines, the LHCII trimer complex contained equal amounts of some regulatory proteins involved in PSII biogenesis and repair (i.e. OHP2, PSB27), and the OHP1 protein showed significantly higher accumulation in both nsi mutant lines as compared to wt and $\sin 7$ (Table 3). It should be noted, however, that at least part of the detected proteins in each band may be contaminants or protein aggregates co-migrating with the true components of the LHCII trimers. Moreover, our previous analysis has evidenced acetylation of the LHCB2.2 protein in the LHCII trimers of both $\sin 7$ and the wild type in two to three replicates, while it was not detected at all in $n s i-1$ and only once in $n s i-2$ (Koskela et al. 2018).

In contrast to digitonin, DM effectively solubilizes the entire thylakoid membrane, including grana stacks and it has been shown that with DM, larger amount of the LHCII trimers disconnect from the photosystems when compared to digitonin (Rantala et al. 2017). MS analysis of the LHCII trimer from the DM samples (defined in Fig. 2b) demonstrated that LHCB2.2, LHCB1.4 and LHCB1.5 were the most abundant LHCB proteins (also when grana stacks were included), and that the relative portion of LHCB 1.4 (compared to LHCB3) in LHCII trimers was increased tenfold as compared to digitonin-solubilized samples (Table 4). These complexes also contained significant amounts of LHCB1.1/2, which was absent in the LHCII trimers of the digitonin-solubilized thylakoids. LHCB4.1, LHCB4.2, LHCB5, and LHCB6 were much more abundant in whole thylakoids than in the non-appressed thylakoids, in agreement with their preferential localization of PSII complexes enriched in grana cores. In contrast, LHCB 1.3 was solely detected in digitonin-solubilized thylakoids, suggesting a preferential localization in stroma thylakoids. Additionally, 
Table 4 Quantitative proteome data analysis according to iBAQ values of the proteins identified within the LHCII trimer bands excised from lpBN-PAGE gels

\begin{tabular}{|c|c|c|c|c|c|c|}
\hline \multicolumn{2}{|c|}{ Proteins/protein groups } & \multirow[t]{2}{*}{ Subunit of } & \multicolumn{4}{|l|}{ Genotype } \\
\hline Locus/loci & Name(s) & & wt & $n s i-1$ & $n s i-2$ & $\sin 7$ \\
\hline AT2G05070 & LHCB 2.2 & LHCII & $9.86( \pm 1.53)$ & $8.50( \pm 2.91)$ & $10.96( \pm 1.11)$ & $13.47( \pm 4.69)$ \\
\hline AT2G34430 & LHCB 1.4 & LHCII & $5.28( \pm 1.20)$ & $4.78( \pm 2.36)$ & $3.06( \pm 0.00)$ & $2.25( \pm 1.00)$ \\
\hline AT2G34420 & LHCB1.5 & LHCII & $2.22( \pm 0.00)$ & $2.17( \pm 1.04)$ & $2.77( \pm 0.00)$ & $4.40( \pm 2.60)$ \\
\hline $\begin{array}{l}\text { AT1G29920; } \\
\text { AT1G29910 }\end{array}$ & LHCB 1.1;LHCB1.2 & LHCII & $1.20( \pm 0.00)$ & $1.31( \pm 0.00)$ & $1.02( \pm 0.00)$ & $1.36( \pm 0.00)$ \\
\hline AT5G54270 & LHCB3 & LHCII & 1.00 & 1.00 & 1.00 & 1.00 \\
\hline AT4G10340 & CP26 (LHCB5) & PSII & $0.27( \pm 0.00)$ & $0.14( \pm 0.00)$ & $0.21( \pm 0.00)$ & $0.19( \pm 0.00)$ \\
\hline AT3G08940 & CP29.2 (LHCB4.2) & PSII & $0.18( \pm 0.00)$ & $0.17( \pm 0.00)$ & $0.16( \pm 0.00)$ & $0.62( \pm 0.00)$ \\
\hline ATCG00680 & CP47 (PSBB) & PSII & $0.16( \pm 0.00)$ & $0.15( \pm 0.00)$ & $0.15( \pm 0.00)$ & $0.51( \pm 0.00)$ \\
\hline ATCG00270 & D2 (PSBD) & PSII & $0.14( \pm 0.00)$ & $0.13( \pm 0.00)$ & $0.16( \pm 0.00)$ & $0.17( \pm 0.00)$ \\
\hline AT3G16140 & PSAH-1 & PSI & $0.11( \pm 0.00)$ & $0.09( \pm 0.00)$ & $0.21( \pm 0.00)$ & $0.59( \pm 0.00)$ \\
\hline ATCG00020 & D1 (PSBA) & PSII & $0.10( \pm 0.00)$ & $0.09( \pm 0.00)$ & $0.12( \pm 0.00)$ & $0.16( \pm 0.00)$ \\
\hline AT5G66570 & PSBO-1 & PSII & $0.08( \pm 0.00)$ & $0.08( \pm 0.00)$ & $0.10( \pm 0.00)$ & $0.10( \pm 0.00)$ \\
\hline AT3G47470 & LHCA4 & PSI & $0.08( \pm 0.00)$ & $0.06( \pm 0.00)$ & $0.07( \pm 0.00)$ & $0.17( \pm 0.00)$ \\
\hline AT1G44575 & NPQ4 (PSBS) & aux & $0.07( \pm 0.00)$ & $0.07( \pm 0.00)$ & $0.09( \pm 0.00)$ & $0.06( \pm 0.00)$ \\
\hline AT5G01530 & CP29.1 (LHCB4.1) & PSII & $0.07( \pm 0.00)$ & $0.04( \pm 0.00)$ & $0.05( \pm 0.00)$ & $0.11( \pm 0.00)$ \\
\hline AT1G15820 & CP24 (LHCB6) & PSII & $0.06( \pm 0.00)$ & $0.05( \pm 0.00)$ & $0.05( \pm 0.00)$ & $0.09( \pm 0.00)$ \\
\hline AT1G31330 & PSAF & PSI & $0.06( \pm 0.00)$ & $0.05( \pm 0.00)$ & $0.05( \pm 0.00)$ & $0.05( \pm 0.00)$ \\
\hline ATCG00340 & PSAB & PSI & $0.05( \pm 0.00)$ & $0.04( \pm 0.00)$ & $0.06( \pm 0.00)$ & $0.14( \pm 0.00)$ \\
\hline AT5G47110 & LIL3.2 & aux & $0.05( \pm 0.00)$ & $0.05( \pm 0.00)$ & $0.07( \pm 0.00)$ & $0.06( \pm 0.00)$ \\
\hline AT4G17600 & LIL3.1 & aux & $0.05( \pm 0.00)$ & $0.04( \pm 0.00)$ & $0.06( \pm 0.00)$ & $0.05( \pm 0.00)$ \\
\hline AT1G61520 & LHCA3 & PSI & $0.05( \pm 0.00)$ & $0.05( \pm 0.00)$ & $0.05( \pm 0.00)$ & $0.08( \pm 0.00)$ \\
\hline AT1G34000 & OHP2 & aux & $0.05( \pm 0.00)$ & $0.06( \pm 0.00)$ & $0.10( \pm 0.00)$ & $0.04( \pm 0.00)$ \\
\hline ATCG00280 & CP43 (PSBC) & PSII & $0.04( \pm 0.00)$ & $0.04( \pm 0.00)$ & $0.04( \pm 0.00)$ & $0.10( \pm 0.00)$ \\
\hline AT3G54890 & LHCA1 & PSI & $0.04( \pm 0.00)$ & $0.03( \pm 0.00)$ & $0.03( \pm 0.00)$ & $0.03( \pm 0.00)$ \\
\hline AT4G28750 & PSAE-1 & PSI & $0.03( \pm 0.00)$ & $0.02( \pm 0.00)$ & $0.04( \pm 0.00)$ & $0.04( \pm 0.00)$ \\
\hline AT3G61470 & LHCA2 & PSI & $0.03( \pm 0.00)$ & $0.02( \pm 0.00)$ & $0.02( \pm 0.00)$ & $0.05( \pm 0.00)$ \\
\hline AT5G02120 & OHP1 & aux & $0.03( \pm 0.00)$ & $0.04( \pm 0.00)$ & $0.05( \pm 0.00)$ & $0.03( \pm 0.00)$ \\
\hline AT4G12800 & PSAL & PSI & $0.02( \pm 0.00)$ & $0.02( \pm 0.00)$ & $0.03( \pm 0.00)$ & $0.08( \pm 0.00)$ \\
\hline AT5G64040 & PSAN & PSI & $0.02( \pm 0.00)$ & $0.02( \pm 0.00)$ & $0.04( \pm 0.00)$ & $0.02( \pm 0.00)$ \\
\hline ATCG00350 & PSAA & PSI & $0.02( \pm 0.00)$ & $0.02( \pm 0.00)$ & $0.03( \pm 0.00)$ & $0.06( \pm 0.00)$ \\
\hline AT1G30380 & PSAK & PSI & $0.02( \pm 0.00)$ & $0.02( \pm 0.00)$ & $0.03( \pm 0.00)$ & $0.12( \pm 0.00)$ \\
\hline AT4G02770 & PSAD-1 & PSI & $0.02( \pm 0.00)$ & $0.01( \pm 0.00)$ & $0.02( \pm 0.00)$ & $0.04( \pm 0.00)$ \\
\hline AT1G79040 & PSBR & PSII & $0.02( \pm 0.00)$ & $0.02( \pm 0.00)$ & $0.01( \pm 0.00)$ & $0.01( \pm 0.00)$ \\
\hline AT1G52230 & PSAH-2 & PSI & $0.01( \pm 0.00)$ & $0.01( \pm 0.00)$ & $0.01( \pm 0.00)$ & $0.03( \pm 0.00)$ \\
\hline AT1G08380 & PSAO & PSI & $0.01^{\mathrm{a}}$ & $0.01^{\mathrm{a}}$ & $0.02^{\mathrm{a}}$ & $0.04^{\mathrm{a}}$ \\
\hline AT3G50820 & PSAO-2 & PSI & $0.01( \pm 0.00)$ & $0.01( \pm 0.00)$ & $0.01( \pm 0.00)$ & $0.01( \pm 0.00)$ \\
\hline AT4G21280 & PSBQ-1 & PSII & $0.01( \pm 0.00)$ & $0.01( \pm 0.00)$ & $0.01( \pm 0.00)$ & $0.01( \pm 0.00)$ \\
\hline
\end{tabular}

Thylakoid membranes were solubilized with dodecyl maltoside, complexes separated using lpBN-PAGE and selected bands excised for LC-MS/ MS analysis. Annotated PSI, PSII and LHC subunits as well as auxiliary PS (aux.) components are presented. The quantity of each protein was normalized to LHCB3, and the relative protein abundances between genotypes were compared using ANOVA/Brown-Forsythe. Post-hoc analysis of significantly different protein groups (ANOVA $p<0.05$ ) was performed using Tukey-HSD. The average relative abundances of detected proteins ( \pm standard deviation) are shown $(n=3 ; N=12)$ ${ }^{\mathrm{a}} n=1$

LIL3.1 and LIL3.2 proteins, which are required for chlorophyll and tocopherol biosynthesis (Tanaka et al. 2010; Lohscheider et al. 2015), were detected in the LHCII trimers in whole thylakoids. Some differences were also found in the accumulation of PSI and PSII proteins in the LHC trimers between the DM- and digitonin-solubilized thylakoids. 
PSAH-1, PSAO-2, PSBQ-1 and PSBR were only detected in whole thylakoids, while PSAP, PSAR and PSBL were detected only in grana margins and stroma lamellae. In the DM LHCII trimer, PSBQ-2 was more abundant in $n s i-2$ than in wt (Table 4).

We also analyzed the lysine acetylation status of the proteins present in the PSI/PSII band and LHCII trimers (Tables S1 and S2). It should be noted, however, that the low concentration of proteins eluted from the lpBN gel bands prohibited the usual antibody-based enrichment of acetylated peptides. This limitation resulted in markedly lower number of detected acetylated peptides as compared to total acetylome analysis (Koskela et al. 2018). Nevertheless, several PSI, PSII, LHCI and LHCII subunits, including PSAA/PSAB, PSAD, PSAH, PSBO2, LHCA1, LHCB1 and LHCB2, were found to be lysine-acetylated (Tables S1 and $\mathrm{S} 2$ ).

\section{Discussion}

Plants are well equipped for acclimation to major fluctuations they experience in their natural environments. For instance, changes in ambient illumination require safe quenching and/or rapid adjustments in the distribution of absorbed energy between the photosystems. The LHCII complex is in the center of these adjustments, as it may either absorb or quench light energy, and regulate energy distribution by associating with either PSI or PSII (Rochaix 2014). The $\sin 7$ mutants, lacking LHCII phosphorylation, show retarded growth when exposed to fluctuating light conditions, which indicates an important role for LHCII phosphorylation and state transitions in response to environmental changes (Bellafiore et al. 2005; Tikkanen et al. 2010). Our results show delayed growth and reduced accumulation of chlorophyll in the $\operatorname{stn} 7$ and the two nsi mutants under fluctuating light (Fig. 1a-c), supporting the view that state transitions are indeed required to adapt to fluctuating light conditions. In line with the reduced growth, and in contrast to those of wt, the PSII capacity $\left(F_{\mathrm{V}} / F_{\mathrm{M}}\right)$ and yield (Y(II)) of all mutant lines showed a substantial decrease with fluctuating light treatment (Fig. 1e,f). The drastic decrease of NPQ detected in both $n s i$ lines but not in $\sin 7$ under fluctuating light as compared to standard light conditions indicates that the capacity of the nsi plants to quench absorbed light energy is severely disturbed (Fig. 1g). In our previous study, we have shown that under high light illumination ( $>200 \mu \mathrm{mol}$ photons $\mathrm{m}^{-2} \mathrm{~s}^{-1}$ ) the $n$ si plants grown under standard conditions exhibit higher steady-state NPQ than wt (Koskela et al. 2018). Future studies are needed to pinpoint the reason behind the differential NPQ capacity of $n s i$ plants: is it a consequence of decreased formation of lumenal $\Delta \mathrm{pH}$, differential accumulation of PSBS or xanthophylls at the thylakoids (Derks et al. 2015), or decreased Lys acetylation status of some LHC proteins detected in nsi mutants (Koskela et al. 2018)?

Application of DM and digitonin solubilization followed by lpBN gel electrophoresis and LC-MS/MS analysis allowed us to conclude that PSII complexes are indeed enriched in grana stacks as compared to grana margins and stroma thylakoids, which is in line with the dogma of lateral heterogeneity of thylakoids (Albertsson 2001; Tikkanen et al. 2012; Pribil et al. 2014). The most abundant LHCII proteins in the free LHCII trimers present in the nonappressed thylakoids were LHCB2.2, LHCB1.5, LHCB3 and LHCB1.4 (Table 3). It has been shown that the L-LHCII trimers are enriched with LHCB1.4, LHCB1.5 and LHCB2, while LHCB3 (and LHCB1.4) proteins are abundant in M-LHCII, which contain very little LHCB1.5 and LHCB2 (Galka et al. 2012). S-LHCII trimers, in turn, are enriched with LHCB1.1-3 (Galka et al. 2012). As only relatively low amount of LHCB1.1-3 protein were detected in the LHCII trimer band, it seems conceivable that the LHCII trimer mainly represents the L-LHCII trimers accompanied with some M-LHCII trimer (Table 3). The absence (or low quantity) of LHCB1.1-3 in the LHCII trimer of grana margins and stroma lamellae (Table 3), together with the fact that LHCB1.1-2 and LHCB2 proteins were more abundant than LHCB1.4 and LHCB1.5 in the PSI/PSII complex (Table 1), suggests that the S-LHCII trimers have remained attached to PSII, which is in line with previous findings (Boekema et al. 1999; Kouril et al. 2012; Rantala et al. 2017). M-LHCII trimers appeared to be more evenly distributed between the PSII-bound pool and LHCII trimer pool.

Under standard growth conditions, the L-LHCII trimers in the grana margins (and stroma lamellae) of wt plants are associated with PSI forming the state transition complex, while such complex formation and consequent energy transfer is not detected in stn 7 and nsi (Figs. 2 and 3). Intriguingly, no differences could be detected in the composition and abundance of LHCII proteins in the LHCII trimer band between the different plant lines (Tables 3 and 4). This result may suggest that the L-LHCII trimers in $\sin 7$ and $n s i$ remain tightly attached to the PSII-LHCII (even upon strong phosphorylation, as in $n s i$ ), because if the PSII-LHCII interaction was weakened, higher accumulation of LHCB2 and LHCB1.4-5 in the LHCII trimers in $s t n 7$ and $n s i$ than in wt would be expected. However, as the pool of L-LHCII capable of associating with PSI is very small (Järvi et al. 2011; Grieco et al. 2015), this effect may be masked by the high total quantity of LHCII. Moreover, it has been shown that the total content of LHCB1 is markedly downregulated in $\sin 7$, with a concomitant increase in the amount of LHCB2 (Tikkanen et al. 2006). The decrease in the LHCB1 amount in $\sin 7$ was evident in the PSI/PSII complex of non-appressed thylakoid membranes, but we did not detect 
an increase in LHCB2 content (Table 2). Moreover, as the composition of LHCII proteins in the LHCII trimer band did not differ between the lines, it is possible that the shift in LHCB1/LHCB2 ratio concerns mainly the LHCII trimers that are forming the high molecular weight PSII-LHCII supercomplexes and PSI-PSII-LHCII megacomplex (Suorsa et al. 2015; Rantala et al. 2017), which were not investigated in this study.

The OHP2 protein was present in the PSI/PSII complexes of grana margins and stroma lamellae, but not in those of grana stacks (Supplemental Tables S1 and S2). This is in agreement with the recently resolved role of OHP1 (and OHP2) in the early stages of PSII de novo assembly and repair under high light conditions (Myouga et al. 2018; Li et al. 2019). OHP1 and OHP2 proteins belong to the light harvesting-like protein family, which contain a chlorophyllbinding domain and one transmembrane helix (Adamska et al. 1999; Rochaix and Bassi 2019). Intriguingly, OHP1 protein amount was significantly higher in the LHCII trimers of non-appressed thylakoids in both nsi mutants as compared to wt and $\sin 7$ (Table 1). Further studies are needed to clarify the role of OHP1 in the nsi plants. Another marked difference between the plant lines was the decreased accumulation of PSBO proteins in the PSI/PSII complexes of non-appressed thylakoids in $n s i$ as compared to wt and $\sin 7$ (Table 1). PSBO-1 and PSBO-2 genes encode the extrinsic PSBO proteins in the PSII oxygen-evolving complex (De Las Rivas et al. 2004), and play an important role in stabilization of the $\mathrm{Mn}_{4} \mathrm{Ca}$-cluster (Miyao and Murata 1984). PSBO-1 is the major isoform in Arabidopsis required for the optimal lateral migration of PSII complexes between the grana and stroma lamellae in response to high light illumination (Allahverdiyeva et al. 2009). As the total acetylome analysis showed decreased acetylation of another OEC protein, i.e. PSBP in the $n s i$ mutants as compared to wt (Koskela et al. 2018), OEC might play a role in the dynamic formation of protein megacomplexes at the thylakoid membrane in response to changes in ambient illumination. Indeed, PSBO is required for binding of PSBP to PSII (Kavelaki and Ghanotakis 1991), while PSBQ stabilizes the association between PSBP and PSBO (Kakiuchi et al. 2012; Bricker et al. 2012; Allahverdiyeva et al. 2013). It has also been shown that deficiency in the OEC results in impaired kinetics of state transitions (Allahverdiyeva et al. 2013). It is important to point out that the overall protein abundances of the proteins, which we found differentially accumulated in the PSI complexes and PSII dimers (PSI/PSII) complex and LHCII trimer in $n s i$ were not found altered in their total protein abundance compared to wt (see Supplemental dataset 2; Koskela et al. 2018). Hence, we hypothesize that chemical modifications of these proteins, such as lysine acetylation, might alter their ability to engage in protein-protein interactions and to reside in specific complexes.
Taken together, our results imply that despite the major molecular phenotype (lack of interaction between PSI and LHCII and thus state transitions) in the $n s i$ and $s t n 7$ mutants, only minor changes could be detected in the composition of the involved protein complexes. Therefore, different kinds of post-translational modifications, such as acetylation and phosphorylation, appear to control the dynamic structure of thylakoid membrane and to regulate the formation of protein complexes in response to environmental cues. Future studies will reveal the impact of OEC on complex formation and resolve the putative role of OHP1 in the nsi mutants.

Acknowledgements Open access funding provided by University of Turku (UTU) including Turku University Central Hospital. Dr. Marjaana Rantala is thanked for invaluable help during the preparation of the manuscript. This study was financially supported by Academy of Finland (316472 and 307335) for M.M.K., A.I and P.M., Doctoral Programme in Molecular Life Sciences at the University of Turku (M.M.K. and A.I.), the Deutscher Akademischer Austauschdienst (financed by Bundesministerium für Bildung und Forschung (BMBF), DAAD project number 57405300) for I.F., "Professorinnenprogramm" of the University of Muenster for A.B., I.F. and the Deutsche Forschungsgemeinschaft (FI 1655/3-1, INST 211/744-1 FUGG) for I.F. HHK received funding via an NSF Career Award (IOS-1553506) and the 3rd call ERA-CAPS call via the NSF PGRP program. This project was carried out within the ERA-CAPS Research Programme "KatNat".

\section{Compliance with ethical standards}

Conflict of interest: The authors declare that they have no conflict of interest.

Open Access This article is licensed under a Creative Commons Attribution 4.0 International License, which permits use, sharing, adaptation, distribution and reproduction in any medium or format, as long as you give appropriate credit to the original author(s) and the source, provide a link to the Creative Commons licence, and indicate if changes were made. The images or other third party material in this article are included in the article's Creative Commons licence, unless indicated otherwise in a credit line to the material. If material is not included in the article's Creative Commons licence and your intended use is not permitted by statutory regulation or exceeds the permitted use, you will need to obtain permission directly from the copyright holder. To view a copy of this licence, visit http://creativecommons.org/licenses/by/4.0/.

\section{References}

Adamska I, Roobol-Boza M, Lindahl M, Andersson B (1999) Isolation of pigment-binding early light-inducible proteins from pea. Eur J Biochem 260:453-460

Albertsson P (2001) A quantitative model of the domain structure of the photosynthetic membrane. Trends Plant Sci 6:349-358

Allahverdiyeva Y, Mamedov F, Holmström M, Nurmi M, Lundin B, Styring S, Spetea C, Aro EM (2009) Comparison of the electron transport properties of the psbol and psbo2 mutants of Arabidopsis thaliana. Biochim Biophys Acta 1787:1230-1237

Allahverdiyeva Y, Suorsa M, Rossi F, Pavesi A, Kater MM, Antonacci A, Tadini L, Pribil M, Schneider A, Wanner G, Leister D, Aro EM, Barbato R, Pesaresi P (2013) Arabidopsis plants lacking 
PsbQ and PsbR subunits of the oxygen-evolving complex show altered PSII super-complex organization and short-term adaptive mechanisms. Plant J 75:671-684

Allen JF, Bennett J, Steinback KE, Arntzen CJ (1981) Chloroplast protein phosphorylation couples plastoquinone redox state to distribution of excitation energy between photosystems. Nature 291:25-29

Aro EM, Suorsa M, Rokka A, Allahverdiyeva Y, Paakkarinen V, Saleem A, Battchikova N, Rintamäki E (2005) Dynamics of photosystem II: a proteomic approach to thylakoid protein complexes. J Exp Bot 56:347-356

Bellafiore S, Barneche F, Peltier G, Rochaix JD (2005) State transitions and light adaptation require chloroplast thylakoid protein kinase STN7. Nature 433:892-895

Bennett J (1977) Phosphorylation of chloroplast membrane polypeptides. Nature 269:344-346

Ben-Shem A, Frolow F, Nelson N (2003) Crystal structure of plant photosystem I. Nature 426:630-635

Benson SL, Maheswaran P, Ware MA, Hunter CN, Horton P, Jansson S, Ruban AV, Johnson MP (2015) An intact light harvesting complex I antenna system is required for complete state transitions in Arabidopsis. Nat Plants 1:15176

Boekema EJ, van Roon H, Calkoen F, Bassi R, Dekker JP (1999) Multiple types of association of photosystem II and its light-harvesting antenna in partially solubilized photosystem II membranes. Biochemistry 38:2233-2239

Boekema EJ, Jensen PE, Schlodder E, van Breemen JF, van Roon H, Scheller HV, Dekker JP (2001) Green plant photosystem I binds light-harvesting complex I on one side of the complex. Biochemistry 40:1029-1036

Bonaventura C, Myers J (1969) Fluorescence and oxygen evolution from Chlorella pyrenoidosa. Biochim Biophys Acta 189:366-383

Bricker TM, Roose JL, Fagerlund RD, Frankel LK, Eaton-Rye JJ (2012) The extrinsic proteins of Photosystem II. Biochim Biophys Acta 1817:121-142

Buchanan BB, Balmer Y (2005) Redox regulation: a broadening horizon. Annu Rev Plant Biol 56:187-220

Cox J, Mann M (2008) MaxQuant enables high peptide identification rates, individualized p.p.b.-range mass accuracies and proteomewide protein quantification. Nat Biotechnol 26:1367-1372

Crepin A, Caffarri S (2015) The specific localizations of phosphorylated Lhcb1 and Lhcb2 isoforms reveal the role of Lhcb2 in the formation of the PSI-LHCII supercomplex in Arabidopsis during state transitions. Biochim Biophys Acta 1847:1539-1548

Danielsson R, Suorsa M, Paakkarinen V, Albertsson PA, Styring S, Aro EM, Mamedov F (2006) Dimeric and monomeric organization of photosystem II. Distribution of five distinct complexes in the different domains of the thylakoid membrane. J Biol Chem 281:14241-14249

De Las Rivas J, Balsera M, Barber J (2004) Evolution of oxygenic photosynthesis: genome-wide analysis of the OEC extrinsic proteins. Trends Plant Sci 9:18-25

Dekker JP, Boekema EJ (2005) Supramolecular organization of thylakoid membrane proteins in green plants. Biochim Biophys Acta 1706:12-39

Depège N, Bellafiore S, Rochaix J (2003) Role of chloroplast protein kinase Stt7 in LHCII phosphorylation and state transition in Chlamydomonas. Science 299:1572-1575

Derks A, Schaven K, Bruce D (2015) Diverse mechanisms for photoprotection in photosynthesis. Dynamicregulation of photosystem II excitation in response to rapidenvironmental change. Biocim Biophys Acta 1847:468-485

Galka P, Santabarbara S, Khuong TT, Degand H, Morsomme P, Jennings RC, Boekema EJ, Caffarri S (2012) Functional analyses of the plant photosystem I-light-harvesting complex II supercomplex reveal that light-harvesting complex II loosely bound to photosystem II is a very efficient antenna for photosystem I in state II. Plant Cell 24:2963-2978

Grieco M, Tikkanen M, Paakkarinen V, Kangasjärvi S, Aro EM (2012) Steady-state phosphorylation of light-harvesting complex II proteins preserves photosystem I under fluctuating white light. Plant Physiol 160:1896-1910

Grieco M, Suorsa M, Jajoo A, Tikkanen M, Aro EM (2015) Lightharvesting II antenna trimers connect energetically the entire photosynthetic machinery-including both photosystems II and I. Biochim Biophys Acta 1847:607-619

Jansson S (1999) A guide to the Lhc genes and their relatives in Arabidopsis. Trends Plant Sci 4:236-240

Järvi S, Suorsa M, Paakkarinen V, Aro EM (2011) Optimized native gel systems for separation of thylakoid protein complexes: novel super- and mega-complexes. Biochemistry J 439:207-214

Kakiuchi S, Uno C, Ido K, Nishimura T, Noguchi T, Ifuku K, Sato F (2012) The PsbQ protein stabilizes the functional binding of the PsbP protein to photosystem II in higher plants. Biochim Biophys Acta 1817:1346-1351

Kavelaki K, Ghanotakis DF (1991) Effect of the manganese complex on the binding of the extrinsic proteins $(17,23$ and $33 \mathrm{kDa})$ of Photosystem II. Photosynth Res 29:149-155

Koochak H, Puthiyaveetil S, Mullendore DL, Li M, Kirchhoff H (2019) The structural and functional domains of plant thylakoid membranes. Plant J 97:412-429

Koskela MM, Brünje A, Ivanauskaite A, Grabsztunowicz M, Lassowskat I, Neumann U, Dinh TV, Sindlinger J, Schwarzer D, Wirtz M, Tyystjärvi E, Finkemeier I, Mulo P (2018) Chloroplast acetyltransferase NSI is required for state transitions in Arabidopsis thaliana. Plant Cell 30:1695-1709

Kouril R, Zygadlo A, Arteni AA, de Wit CD, Dekker JP, Jensen PE, Scheller HV, Boekema EJ (2005) Structural characterization of a complex of photosystem I and light-harvesting complex II of Arabidopsis thaliana. Biochemistry 44:10935-10940

Kouril R, Dekker JP, Boekema EJ (2012) Supramolecular organization of photosystem II in green plants. Biochim Biophys Acta 1817:2-12

Kyle DJ, Staehelin LA, Arntzen CJ (1983) Lateral mobility of the lightharvesting complex in chloroplast membranes controls excitation energy distribution in higher plants. Arch Biochem Biophys 222:527-541

Li Y, Liu B, Zhang J, Kong F, Zhang L, Meng H, Li W, Rochaix JD, Li D, Peng L (2019) OHP1, OHP2, and HCF244 form a transient functional complex with the Photosystem II reaction center. Plant Physiol 179:195-208

Lohscheider JN, Rojas-Stutz MC, Rothbart M, Andersson U, Funck D, Mendgen K, Grimm B, Adamska I (2015) Altered levels of LIL3 isoforms in Arabidopsis lead to disturbed pigment-protein assembly and chlorophyll synthesis, chlorotic phenotype and impaired photosynthetic performance. Plant Cell Environ 38:2115-2127

Longoni P, Douchi D, Cariti F, Fucile G, Goldschmidt-Clermont M (2015) Phosphorylation of the light-harvesting complex II isoform Lhcb2 is central to state transitions. Plant Physiol 169:2874-2883

Lunde C, Jensen PE, Haldrup A, Knoetzel J, Scheller HV (2000) The PSI-H subunit of photosystem I is essential for state transitions in plant photosynthesis. Nature 408:613-615

Miyao M, Murata N (1984) Effect of urea on photosystem II particles: evidence for an essential role of the 33 kilodalton polypeptide in photosynthetic oxygen evolution. Biochim Biophys Acta 765:253-257

Morgan MJ, Lehmann M, Schwarzlander M, Baxter CJ, SienkiewiczPorzucek A, Williams TC, Schauer N, Fernie AR, Fricker MD, Ratcliffe RG, Sweetlove LJ, Finkemeier I (2008) Decrease in manganese superoxide dismutase leads to reduced root growth 
and affects tricarboxylic acid cycle flux and mitochondrial redox homeostasis. Plant Physiol 147:101-114

Murata N (1969) Control of excitation transfer in photosynthesis. I. Light-induced change of chlorophyll a fluorescence in Porphyridium cruentum. Biochim Biophys Acta 172:242-251

Myouga F, Takahashi K, Tanaka R, Nagata N, Kiss AZ, Funk C, Nomura Y, Nakagami H, Jansson S, Shinozaki K (2018) Stable accumulation of Photosystem II requires One-Helix Protein1 (OHP1) of the light harvesting-like family. Plant Physiol 176:2277-2291

Pan X, Ma J, Su X, Cao P, Chang W, Liu Z, Zhang X, Li M (2018) Structure of the maize photosystem I supercomplex with lightharvesting complexes I and II. Science 360:1109-1113

Pesaresi P, Hertle A, Pribil M, Kleine T, Wagner R, Strissel H, Ihnatowicz A, Bonardi V, Scharfenberg M, Schneider A, Pfannschmidt T, Leister D (2009) Arabidopsis STN7 kinase provides a link between short- and long-term photosynthetic acclimation. Plant Cell 21:2402-2423

Pietrzykowska M, Suorsa M, Semchonok DA, Tikkanen M, Boekema EJ, Aro EM, Jansson S (2014) The light-harvesting chlorophyll $\mathrm{a} / \mathrm{b}$ binding proteins Lhcb1 and Lhcb2 play complementary roles during state transitions in Arabidopsis. Plant Cell 26:3646-3660

Porra RJ, Thompson WA, Kriedemann PE (1989) Determination of accurate extinction coefficients and simultaneous-equations for assaying chlorophyll-A and chlorophyll-B extracted with 4 different solvents-verification of the concentration of chlorophyll standards by atomic-absorption spectroscopy. Biochim Biophys Acta 975:384-394

Pribil M, Pesaresi P, Hertle A, Barbato R, Leister D (2010) Role of plastid protein phosphatase TAP38 in LHCII dephosphorylation and thylakoid electron flow. PLoS Biol 8:e1000288

Pribil M, Labs M, Leister D (2014) Structure and dynamics of thylakoids in land plants. J Exp Bot 65:1955-1972

Puthiyaveetil S, Ibrahim IM, Jelicic B, Tomasic A, Fulgosi H, Allen JF (2010) Transcriptional control of photosynthesis genes: the evolutionarily conserved regulatory mechanism in plastid genome function. Genome Biol Evol 2:888-896

Puthiyaveetil S, Ibrahim IM, Allen JF (2012) Oxidation-reduction signalling components in regulatory pathways of state transitions and photosystem stoichiometry adjustment in chloroplasts. Plant Cell Environ 35:347-359

Rantala M, Tikkanen M, Aro EM (2017) Proteomic characterization of hierarchical megacomplex formation in Arabidopsis thylakoid membrane. Plant J 92:951-962

Rochaix JD (2014) Regulation and dynamics of the light-harvesting system. Annu Rev Plant Biol 65:287-309

Rochaix JD, Bassi R (2019) LHC-like proteins involved in stress responses and biogenesis/repair of the photosynthetic apparatus. Biochem J 476:581-593

Schneider D, Lopez LS, Li M, Crawford JD, Kirchhoff H, Kunz H-H (2019) Fluctuating light experiments and semi-automated plant phenotyping enabled by self-built growth racks and simple upgrades to the IMAGING-PAM. Plant Methods 15:156

Schöttler MA, Albus CA, Bock R (2011) Photosystem I: its biogenesis and function in higher plants. J Plant Physiol 168:1452-1461
Schwanhäusser B, Busse D, Li N, Dittmar G, Schuchhardt J, Wolf J, Chen W, Selbach M (2011) Global quantification of mammalian gene expression control. Nature 473:337-342

Shapiguzov A, Ingelsson B, Samol I, Andres C, Kessler F, Rochaix JD, Vener AV, Goldschmidt-Clermont M (2010) The PPH1 phosphatase is specifically involved in LHCII dephosphorylation and state transitions in Arabidopsis. Proc Natl Acad Sci USA 107:4782-4787

Steiner S, Dietzel L, Schröter Y, Fey V, Wagner R, Pfannschmidt T (2009) The role of phosphorylation in redox regulation of photosynthesis genes $p s a A$ and $p s b A$ during photosynthetic acclimation of mustard. Mol Plant 2:416-429

Suorsa M, Rantala M, Danielsson R, Järvi S, Paakkarinen V, Schröder WP, Styring S, Mamedov F, Aro EM (2014) Dark-adapted spinach thylakoid protein heterogeneity offers insights into the photosystem II repair cycle. Biochim Biophys Acta 1837:1463-1471

Suorsa M, Rantala M, Mamedov F, Lespinasse M, Trotta A, Grieco M, Vuorio E, Tikkanen M, Järvi S, Aro EM (2015) Light acclimation involves dynamic re-organization of the pigment-protein megacomplexes in non-appressed thylakoid domains. Plant $\mathrm{J}$ $84: 360-373$

Tanaka R, Rothbart M, Oka S, Takabayashi A, Takahashi K, Shibata M, Myouga F, Motohashi R, Shinozaki K, Grimm B, Tanaka A (2010) LIL3, a light-harvesting-like protein, plays an essential role in chlorophyll and tocopherol biosynthesis. Proc Natl Acad Sci USA 107:16721-16725

Tikkanen M, Piippo M, Suorsa M, Sirpiö S, Mulo P, Vainonen J, Vener AV, Allahverdiyeva Y, Aro EM (2006) State transitions revisited-a buffering system for dynamic low light acclimation of Arabidopsis. Plant Mol Biol 62:779-793

Tikkanen M, Nurmi M, Kangasjärvi S, Aro EM (2008) Core protein phosphorylation facilitates the repair of photodamaged photosystem II at high light. Biochim Biophys Acta 1777:1432-1437

Tikkanen M, Grieco M, Kangasjärvi S, Aro EM (2010) Thylakoid protein phosphorylation in higher plant chloroplasts optimizes electron transfer under fluctuating light. Plant Physiol 152:723-735

Tikkanen M, Suorsa M, Gollan PJ, Aro EM (2012) Post-genomic insight into thylakoid membrane lateral heterogeneity and redox balance. FEBS Lett 586:2911-2916

Trotta A, Bajwa AA, Mancini I, Paakkarinen V, Pribil M, Aro E-M (2019) The role of phosphorylation dynamics of Curvature Thylakoid 1B in plant thylakoid membranes. Plant Phys 181:1615-1631

Tyanova S, Temu T, Sinitcyn P, Carlson A, Hein MY, Geiger T, Mann M, Cox J (2016) The Perseus computational platform for comprehensive analysis of (prote)omics data. Nat Methods 13:731-740

Wientjes E, van Amerongen H, Croce R (2013) LHCII is an antenna of both photosystems after long-term acclimation. Biochim Biophys Acta 1827:420-426

Publisher's Note Springer Nature remains neutral with regard to jurisdictional claims in published maps and institutional affiliations. 